\title{
Galactic kinematics with RAVE data
}

\section{The distribution of stars towards the Galactic poles}

\author{
L. Veltz ${ }^{1,2,3}$, O. Bienaymé ${ }^{1}$, K. C. Freeman ${ }^{2}$, J. Binney ${ }^{4}$, J. Bland-Hawthorn ${ }^{5}$, B. K. Gibson ${ }^{6}$, G. Gilmore ${ }^{7}$, \\ E. K. Grebel ${ }^{8,9}$, A. Helmi ${ }^{10}$, U. Munari ${ }^{11}$, J. F. Navarro ${ }^{12}$, Q. A. Parker ${ }^{13,14}$, G. M. Seabroke ${ }^{7}$, \\ A. Siebert ${ }^{1,3}$, M. Steinmetz ${ }^{3}$, F. G. Watson ${ }^{13}$, M. Williams ${ }^{2,3}$, R. F. G. Wyse ${ }^{15}$, and T. Zwitter ${ }^{16}$
}

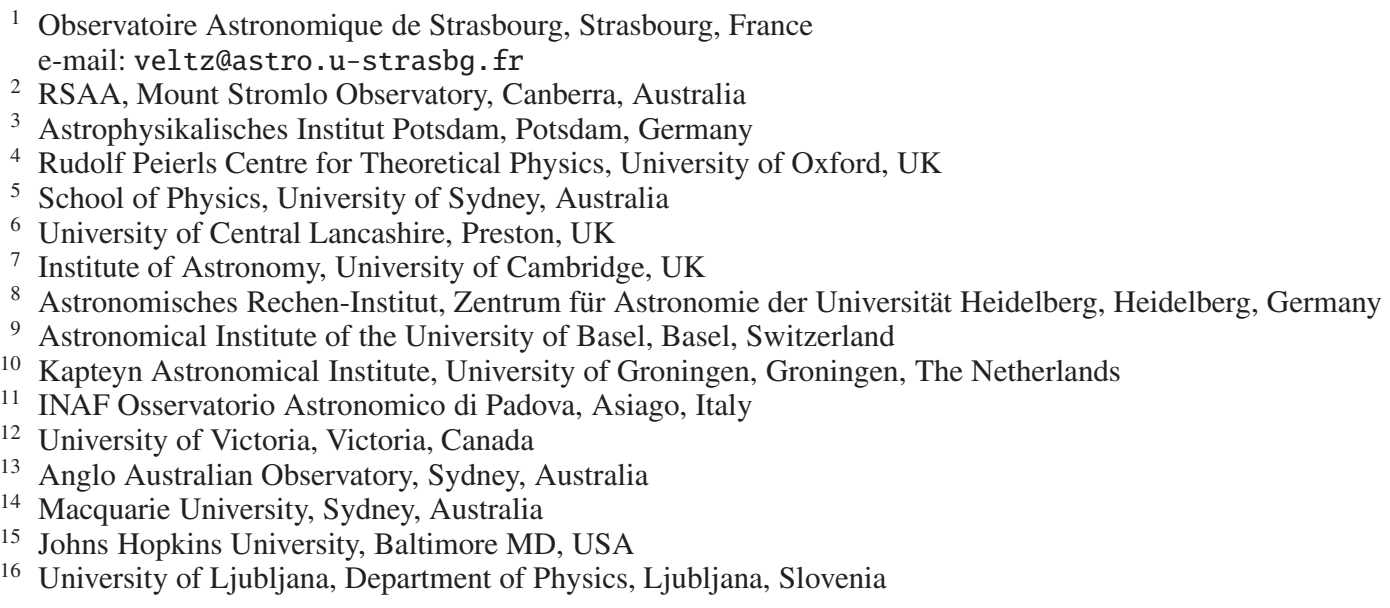

Received 15 December 2006 / Accepted 18 December 2007

\section{ABSTRACT}

\begin{abstract}
We analyze the distribution of $\mathrm{G}$ and $\mathrm{K}$ type stars towards the Galactic poles using RAVE and ELODIE radial velocities, 2MASS photometric star counts, and UCAC2 proper motions. The combination of photometric and 3D kinematic data allows us to disentangle and describe the vertical distribution of dwarfs, sub-giants and giants and their kinematics.

We identify discontinuities within the kinematics and magnitude counts that separate the thin disk, thick disk and a hotter component. The respective scale heights of the thin disk and thick disk are $225 \pm 10 \mathrm{pc}$ and $1048 \pm 36 \mathrm{pc}$. We also constrain the luminosity function and the kinematic distribution function. The existence of a kinematic gap between the thin and thick disks is incompatible with the thick disk having formed from the thin disk by a continuous process, such as scattering of stars by spiral arms or molecular clouds. Other mechanisms of formation of the thick disk such as "created on the spot" or smoothly "accreted" remain compatible with our findings.
\end{abstract}

Key words. stars: kinematics - Galaxy: disk - Galaxy: fundamental parameters - Galaxy: kinematics and dynamics Galaxy: structure

\section{Introduction}

It is now widely accepted that the stellar density distribution perpendicular to the Galactic disk traces at least two stellar components, the thin and the thick disks. The change of slope in the logarithm of the vertical density distributions at $\sim 700 \mathrm{pc}$ (CabreraLavers et al. 2005) or 1500 pc (Gilmore \& Reid 1983) above the Galactic plane is usually explained as the signature of a transition between these two distinct components: the thin and the thick disks. The thick disk is an intermediate stellar population between the thin disk and the stellar halo, and was initially defined with the other stellar populations by combining spatial, kinematic and abundance properties (see a summary of the Vatican conference of 1957 by Blaauw 1995 and Gilmore \& Wyse 1989). Its properties are described in a long series of publications with often diverging characteristics (see the analysis by Gilmore 1985; Ojha 2001; Robin et al. 2003, and also by
Cabrera-Lavers et al. 2005, that give an overview of recent improvements). Majewski (1993) compared a nearly exhaustive list of scenarios that describe many possible formation mechanisms for the thick disk.

In this paper, we attempt to give an answer to the simple but still open questions: are the thin and thick disks really two distinct components? Is there any continuous transition between them? These questions were not fully settled by analysis of star counts by Gilmore \& Reid (1983) and later workers. Other important signatures of the thick disk followed from kinematics: the age-velocity dispersion relation and also the metallicity-velocity dispersion relation. However the identification of a thin-thick discontinuity depends on the authors, due to the serious difficulty of assigning accurate ages to stars (see Edvardsson et al. 1993; Nordström et al. 2004). More recently it was found that the $[\alpha / \mathrm{Fe}]$ versus $[\mathrm{Fe} / \mathrm{H}]$ distribution is related to the kinematics (Fuhrmann 1998; Feltzing et al. 2003; 
Soubiran \& Girard 2005; Brewer \& Carney 2006; Reddy et al. 2006) and provides an effective way to separate stars from the thin and thick disk components. Ages and abundances are important to describe the various disk components and to depict the mechanisms of their formation. A further complication comes from the recent indications of the presence of at least two thick disk components with different density distributions, kinematics and abundances (Gilmore et al. 2002; Soubiran et al. 2003; Wyse et al. 2006).

Many of the recent works favor the presently prevailing scenarios of thick disk formation by the accretion of small satellites, puffing up the early stellar Galactic disk or tidally disrupting the stellar disk (see for example Steinmetz \& Navarro 2002; Abadi et al. 2003; Brook et al. 2004). We note however that chemodynamical models of secular Galactic formation including extended ingredients of stellar formation and gas dynamics can also explain the formation of a thick disk distinct from the thin disk (Samland \& Gerhard 2003; Samland 2004).

In this paper, we use the recent RAVE observations of stellar radial velocities, combined with star counts and proper motions, to recover and model the full 3D distributions of kinematics and densities for nearby stellar populations. In a forthcoming study, metallicities measured from RAVE observations will be included to describe the galactic stellar populations and their history. The description of data is given in Sect. 2, the model in Sect. 3, and the interpretation and results in Sect. 4. Among these results, we identify discontinuities visible both within the density distributions and the kinematic distributions. They allow to define more precisely the transition between the thin and thick stellar Galactic disks.

\section{Observational data}

Three types of data are used to constrain our Galactic model for the stellar kinematics and star counts (the model description is given in Sect. 3): the Two-Micron All-Sky Survey (2MASS PSC; Cutri et al. 2003) magnitudes, the RAVE (Steinmetz et al. 2006) and ELODIE radial velocities, and the UCAC2 (Zacharias et al. 2004) proper motions. Each sample of stars is selected independently of the other, with its own magnitude limit and coverage of sky due to the different source (catalogue) characteristics.

(1) We select 22050 2MASS stars within an 8-degree radius of the South and North Galactic Poles, with $m_{\mathrm{K}}$ magnitudes between 5-15.4. Star count histograms for both Galactic poles are used to constrain the Galactic model.

(2) We select 105170 UCAC2 stars within a radius of 16 degrees of the Galactic poles, with $m_{\mathrm{K}}$ 2MASS magnitudes between 6-14. We adjust the model to fit histograms of the $\mu_{U}$ and $\mu_{V}$ proper motion marginal distributions; the histograms combine stars in 1.0 mag intervals for $m_{\mathrm{K}}=6$ to 9 and 0.2 mag intervals for $m_{\mathrm{K}}=9$ to 14 .

(3) We select 543 RAVE stars (with $m_{\mathrm{K}}$ 2MASS magnitudes from 8.5 to 11.5 ) within a radius of 15 degrees of the SGP. We group them in three histograms according to $m_{\mathrm{K}}$ magnitudes. We complete this radial velocity sample with 392 other similar stars: TYCHO-II stars selected towards the NGP within an area of 720 square degrees, with $B-V$ colors between 0.9-1.1. Their magnitudes are brighter than $m_{\mathrm{K}}=8.5$, they were observed with the ELODIE spectrograph and were initially used to probe the vertical Galactic potential (Bienaymé et al. 2006). All these radial velocity samples play a key role in constraining the vertical velocity distributions of stars and the shape of the velocity ellipsoid.

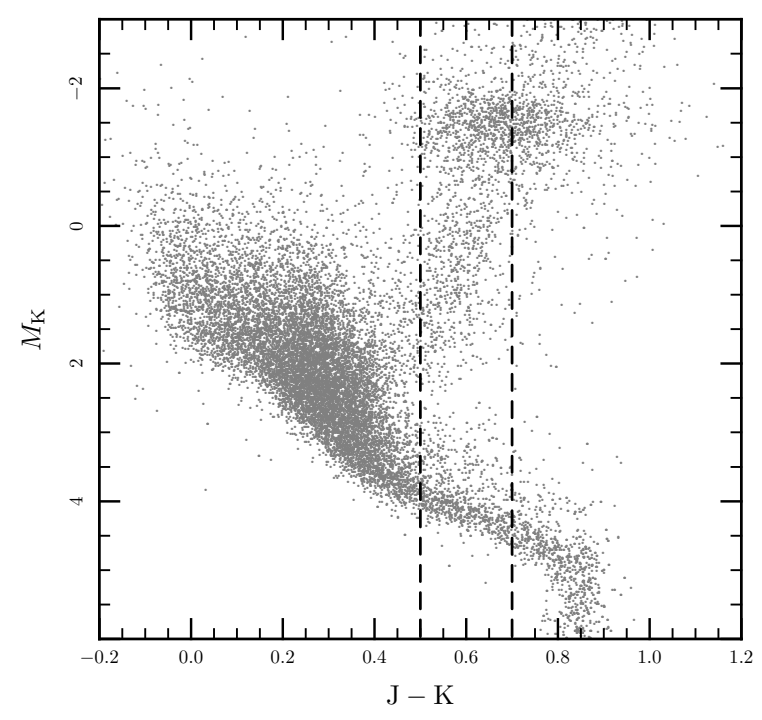

Fig. 1. $M_{K} / J-K$ HR diagram from Hipparcos stars with $\sigma_{\pi} / \pi \leq 0.1$ cross-matched with the 2MASS catalogue. Vertical dashed lines represent our color selection $J-K=[0.5-0.7]$.

\subsection{Data selection}

In this paper, we restrict our analysis to stars near the Galactic poles with $J-K$ colors between $0.5-0.7$ (see Fig. 1). This allows us to recover some Galactic properties, avoiding the coupling with other Galactic parameters that occurs in other Galactic directions (density and kinematic scale lengths, Oort's constants, $\left.R_{0}, V_{0} \ldots\right)$.

The selected $J-K=[0.5-0.7]$ color interval corresponds to K3-K7 dwarfs and G3-K1 giants (Koornneef 1983; Ducati et al. 2001). They may be $\mathrm{G}$ or $\mathrm{K}$ giants within the red clump region (the part of the HR diagram populated by high metallicity He-burning core stars). The absolute magnitudes of red clump stars are well defined: nearby HIPPARCOS clump stars have a mean absolute magnitude $M_{\mathrm{K}}=-1.61$ with a dispersion of $\sim 0.22$ (Alves 2000; see Cannon 1970, for the first proposed use of clump stars as distance indicators, see also Salaris \& Girardi 2002; Girardi et al. 1998, and other references in Cabrera-Lavers et al. 2005). This mean absolute magnitude does not vary significantly with $[\mathrm{Fe} / \mathrm{H}]$ in the abundance range $[-0.6,0]$ (Alves 2000). Studying nearby stars in 13 open clusters and 2 globular clusters, Grocholski \& Sarajedini (2002) find that the mean absolute magnitude of clump stars is not dependent on metallicity when the $[\mathrm{Fe} / \mathrm{H}]$ abundance remains within the interval $[-0.5,0.1]$. Sarajedini (2004) finds that, at metallicity $[\mathrm{Fe} / \mathrm{H}]=-0.76$, the mean absolute magnitude of red clump stars drops to $M_{\mathrm{K}}=-1.28$, a shift of 0.33 mag. Most of the giants with metallicity $[\mathrm{Fe} / \mathrm{H}]$ lower than -0.8 dex are excluded by our color selection from our sample. Hence, we did not model giants of the metal-weak thick disk, first identified by Norris (1985) (see also, Morrison et al. 1990). This represents however only a minor component of the thick disk. Although et al. (2000) find that $\sim 30 \%$ of the stars with $-1>[\mathrm{Fe} / \mathrm{H}]>-1.7$ are thick disk stars, but stars with $[\mathrm{Fe} / \mathrm{H}]<-1$ represent only 1 per cent of the local thick disk stars (Martin \& Morrison 1998).

$\mathrm{K}$ dwarfs within the $J-K=[0.5-0.7]$ color interval also have well defined absolute magnitudes that depend slightly on metallicity and color. We determine their mean absolute magnitude, $M_{\mathrm{K}}=4.15$, from nearby HIPPARCOS stars using color magnitude data provided by Reid (see http: //www-int. stsci.edu/ inr/cmd.html). From Padova isochrones 
(Girardi et al. 2002), we find that the absolute magnitude varies by $0.4 \mathrm{mag}$ when $J-K$ changes from 0.5 to 0.7 . A change of metallicity of $\Delta[\mathrm{Fe} / \mathrm{H}]=0.6$ also changes the magnitude by about 0.3 , in qualitative agreement with observed properties of K dwarfs (Reid 1998, Kotoneva et al. 2002). Thus, we estimate that the dispersion of absolute magnitude of dwarfs in our Galactic pole sample is $\sim 0.2-0.4$.

Another important motivation for selecting the $J-K=[0.5-0.7]$ color interval is the absolute magnitude step of 6 mag between dwarfs and giants. This separation is the reason the magnitude distributions for these two kinds of stars are very different towards the Galactic poles. If giants and dwarfs have the same density distribution in the disk, in the apparent magnitude count, giants will appear before and well separated from dwarfs. Finally we mention a convenient property of the Galactic pole directions: there, the kinematic data are simply related to the cardinal velocities relative to the local standard of rest (LSR). UCAC2 proper motions are nearly parallel to the $U$ and $V$ velocities, and RAVE radial velocities are close to the vertical $W$ velocity component.

\subsection{How accurate is the available data?}

The star magnitudes are taken from the 2MASS survey which is presently the most accurate photometric all sky survey for probing the Galactic stellar populations. Nevertheless, since our color rang is narrow, we have to take care that the photometric errors on $J$ and $K$ do not bias our analysis.

The mean photometric accuracy ranges from 0.02 in $K$ and $J$ at magnitudes $m_{\mathrm{K}}=5.0$, to 0.15 in $K$ and 0.08 in $J$ at magnitudes $m_{\mathrm{K}}=15.4$. The error in $J-K$ is not small considering the size $(\Delta(J-K)=0.2)$ of the analyzed $J-K$ interval, 0.5 to 0.7 . We do not expect, however, that it substantially biases our analysis. For $m_{\mathrm{K}}$ brighter than 10 , the peak of giants is clearly identified in the $J-K$ distribution within the $J-K=[0.5-0.7]$ interval (see Fig. 2 or Fig. 6 from Cabrera-Lavers et al. 2005). This peak vanishes only beyond $m_{\mathrm{K}}=11$. At fainter $K$ mag, the dwarfs dominate and the $J-K$ histogram of colors has a constant slope. This implies that the error in color at faint magnitudes does not affect to first order the star counts.

We find from the shape of the count histograms that, in the direction of the Galactic Pole and with our color selection $J-K=[0.5-0.7]$ the limit of completeness is $m_{\mathrm{K}} \sim 15.5-15.6$. Moreover, the contamination by galaxies must be low within the 2MASS PSC. It is also unlikely that compact or unresolved galaxies are present: according to recent deep $J$ and $K$ photometric counts (see Fig. 15 of Iovino et al. 2005), with our color selection, galaxies contribute only beyond $m_{\mathrm{K}} \sim 16$. We conclude that we have a complete sample of stars for magnitudes from 5.0 to 15.4 in $K$, towards the Galactic poles.

The UCAC2 and RAVE catalogues however are not complete. Making it necessary to scale the proper motions and radial velocities distributions predicted by our model for complete samples. The total number of stars given by the model for the distribution of proper motions (or radial velocity) in a magnitude interval is multiplied by the ratio between the number of stars observed in UCAC2 (or RAVE) divided by the number of stars observed in 2MASS.

Towards the North Galactic Pole (NGP), the error on the UCAC2 proper motions used in our analysis varies from $1 \mathrm{mas} \mathrm{yr}^{-1}$ for the brightest stars to $6 \mathrm{mas} \mathrm{yr}^{-1}$ at $m_{\mathrm{K}}=14$. Towards the South Galactic Pole (SGP), the error distribution looks similar, with the exception of a small fraction of stars with $m_{\mathrm{K}}$ from 11 to 14 having errors around 8 or $13 \mathrm{mas} \mathrm{yr}^{-1}$. The

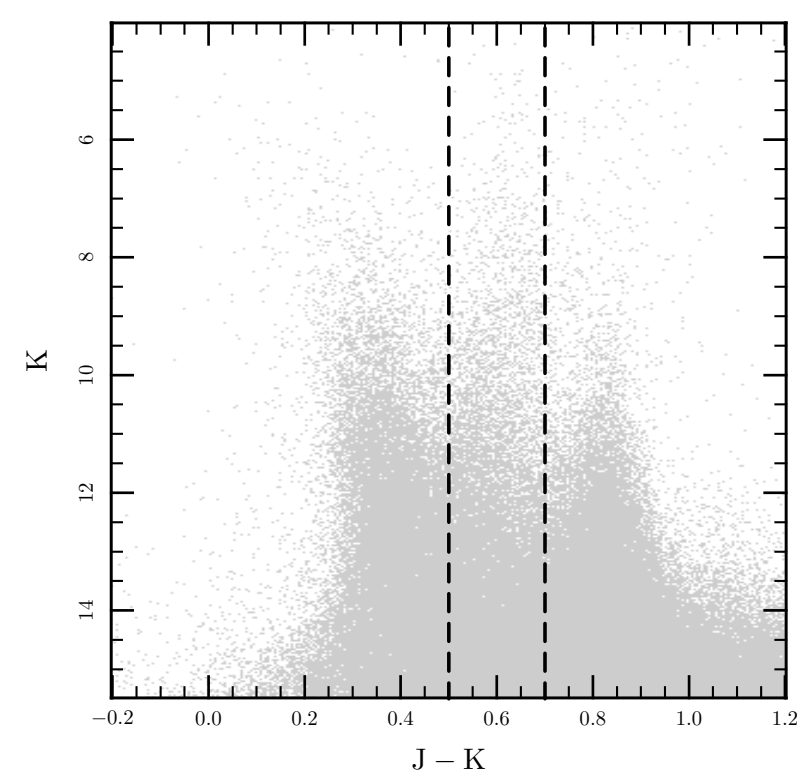

Fig. 2. $K / J-K$ color magnitude diagram obtained with 2MASS stars within a 8 degrees radius around the North Galactic pole. Dashed lines represent the limit of our color selection: $J-K=[0.5,0.7]$.

only noticeable difference between the histograms at the NGP and SGP is that the peak of the proper motion distribution is slightly more flattened at the SGP, for magnitudes $m_{\mathrm{K}}>13$ (see Fig. 6). This difference is related to the different error distributions towards the NGP and SGP.

The analyzed stars are located at distances from $200 \mathrm{pc}$ to

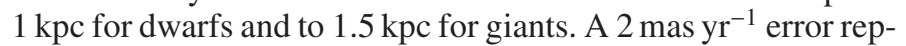
resents $10 \mathrm{~km} \mathrm{~s}^{-1}$ at $1 \mathrm{kpc}$, and $6 \mathrm{mas} \mathrm{yr}^{-1}$, an error of $30 \mathrm{~km} \mathrm{~s}^{-1}$. This can be compared to the $\sigma_{U}$ values for the isothermal components, for instance $\sim 60 \mathrm{~km} \mathrm{~s}^{-1}$ for the thick disk that is the dominant stellar population $1.5 \mathrm{kpc}$ from the plane. Adding the errors in quadrature to the velocity dispersion would modify a real proper motion dispersion of $60 \mathrm{~km} \mathrm{~s}^{-1}$ to an apparent dispersion of $67 \mathrm{~km} \mathrm{~s}^{-1}$. The apparent dispersion would be only $60.8 \mathrm{~km} \mathrm{~s}^{-1}$ if the stars have a 2 mas yr$^{-1}$ accuracy. Therefore, we overestimate the $\sigma_{U}$ dispersion of the thick disk by 5 to 10 percent. This effect is lower for the thin disk components (the stars are closer and their apparent proper motion distributions are broader). We have not yet included the effect of proper motion errors within our model. This error has just an impact of the determination on the velocity dispersions $\sigma_{U}$ and $\sigma_{V}$ and on the ellipsoid axis ratio $\sigma_{U} / \sigma_{W}$ of each stellar disk component, but does not change the determination of vertical velocity dispersions $\sigma_{W}$ which are mainly constrained by the magnitude star count and the radial velocities. Hence, it is not significant in our kinematic decomposition of the Galactic disk.

The accuracy of proper motions can also be gauged from the stability of the peaks of proper motion distributions: comparing $112 \mu_{U}$ and $\mu_{V}$ histograms for different magnitude intervals, we

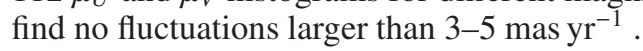

A more complete test is performed by comparing the UCAC2 proper motions (with our $J-K$ color selection) to the recent PM2000 catalogue (Ducourant et al. 2006) in an area of $8 \times 16$ degrees around $\alpha_{2000}=12 \mathrm{~h} 50 \mathrm{~m}, \delta_{2000}=14 \mathrm{deg}$ close to the NGP. PM2000 proper motions are more accurate, with errors from 1 to 4 mas $^{-1}{ }^{-1}$. The mean differences between proper motions from both catalogues versus magnitudes and equatorial coordinates do not show significant shifts, just fluctuations of 
the order of $\sim 0.2$ mas $^{-1} r^{-1}$. We also find that the dispersions of proper motion differences are $\sim 2 \mathrm{mas} \mathrm{yr}^{-1}$ for $m_{\mathrm{K}}<10$, 4 mas yr $^{-1}$ with $m_{\mathrm{K}}=10-13$, and 6 mas yr $^{-1}$ with $m_{\mathrm{K}}=13-14$. These dispersions are dominated by the UCAC2 errors.

From the internal and external error analysis, RAVE radial velocities show a mean accuracy of $2.3 \mathrm{~km} \mathrm{~s}^{-1}$ (Steinmetz et al. 2006). Radial velocities of stars observed with the ELODIE échelle spectrograph are an order of magnitude more accurate. These errors have no impact on the determination of the vertical velocity dispersion of stellar components that ranges from 10 to $50 \mathrm{~km} \mathrm{~s}^{-1}$, but the reduced size of our radial velocity samples towards the poles (about 1000 stars) limits the accuracy achieved in modeling the vertical velocity dispersions.

\section{Model of the stellar Galactic disks}

The basic ingredients of our Galactic model are taken from traditional works on star count and kinematic modeling, for instance see Pritchet (1983); Bahcall (1984); Robin \& Crézé (1986). It is also similar to the recent developments by Girardi et al. (2005) or by Vallenari et al. (2006).

The kinematic modeling is entirely taken from Ratnatunga et al. (1989) and is also similar to Gould's (2003) analysis. Both propose closed-form expressions for velocity projections; the dynamical consistency is similar to Bienaymé et al. (1987) and Robin et al. (2003, 2004).

Our analysis, limited to the Galactic poles, is based on a set of 20 stellar disk components. The distribution function of each component or stellar disk is built from three elementary functions describing the vertical density $\rho_{i}$ (dynamically self consistent with the vertical gravitational potential), the kinematic distribution $f_{i}$ (3D-Gaussians) and the luminosity function $\phi_{i k}$.

We define $\mathcal{N}\left(z, V_{R}, V_{\phi}, V_{z} ; M\right)$ to be the density of stars in the Galactic position-velocity-(absolute magnitude) space

$\mathcal{N}=\sum_{i k} \rho_{i}(z) f_{i}\left(V_{R}, V_{\phi}, V_{z}\right) \phi_{i k}(M)$

the index $i$ differentiates the stellar disk components and the index $k$ the absolute magnitudes used to model the luminosity function.

From this model, we apply the generalized equation of stellar statistics:

$A\left(m, \mu_{l}, \mu_{b}, V_{r}\right)=\int N\left(z, V_{R}, V_{\phi}, V_{z} ; M\right) z^{2} \omega \mathrm{d} z$

to determine the $A(m)$ apparent magnitude star count equation as well as the marginal distributions of both components $\mu_{l}$ and $\mu_{b}$ of proper motions and the distributions of radial velocities for any direction and apparent magnitudes. For the Galactic poles, we define $\mu_{U}$ and $\mu_{V}$ as the proper motion components parallel to the cardinal directions of $U$ and $V$ velocities. For a more general inverse method of the equation of the stellar statistic, see Pichon et al. (2002).

\subsection{The vertical density}

Each stellar disk is modeled with an isothermal velocity distribution, assuming that the vertical density distribution (normalized at $z=0$ ) is given by the relation:

$\rho_{i}(z)=\exp \left(-\Phi(z) / \sigma_{z z, i}^{2}\right)$

where $\Phi(z)$ is the vertical gravitational potential at the solar Galactic position and $\sigma_{z z, i}$ is the vertical velocity dispersion of the considered stellar component $i$. The Sun's position $z_{\odot}$ above the Galactic plane is also used as a model parameter. Such expressions were introduced by Oort (1922), assuming the stationarity of the density distributions. They ensure the consistency between the vertical velocity and density distributions. For the vertical gravitational potential we use the recent determination obtained by Bienaymé et al. (2006) based on the analysis of HIPPARCOS and TYCHO-II red clump giants. The vertical potential is defined at the solar position by:

$$
\Phi(z)=4 \pi G\left(\Sigma_{0}\left(\sqrt{z^{2}+D^{2}}-D\right)+\rho_{\mathrm{eff}} z^{2}\right)
$$

with $\Sigma_{0}=48 M_{\odot} \mathrm{pc}^{-2}, D=800 \mathrm{pc}$ and $\rho_{\text {eff }}=0.07 M_{\odot} \mathrm{pc}^{-3}$.

It is quite similar to the potential determined by Kuijken $\&$ Gilmore (1989) and Holmberg \& Flynn (2004).

\subsection{The kinematic distributions}

The kinematical model is given by shifted 3D Gaussian velocity ellipsoids. The three components of mean streaming motion $(\langle U\rangle,\langle V\rangle,\langle W\rangle)$ and velocity dispersions $\left(\sigma_{R R}, \sigma_{\phi \phi}, \sigma_{z z}\right)$, referred to the cardinal directions of the Galactic coordinate frame, provide a set of six kinematic quantities. The mean stream motion is relative to the LSR. The Sun's velocity $U_{\odot}$ and $W_{\odot}$ are model parameters. We define the $\langle V\rangle$ stream motion as: $\langle V\rangle=-V_{\odot}-V_{\text {lag }}$. We adopt an asymmetric drift proportional to the square of $\sigma_{R R}$ : $V_{\text {lag }}=\sigma_{R R}^{2} / k_{a}$, where the coefficient $k_{a}$ is also a model parameter. We assume null stream motions for the other velocity components, thus $\langle U\rangle=-U_{\odot}$ and $\langle W\rangle=-W_{\odot}$.

For simplicity, we have assumed that the $\sigma_{R R} / \sigma_{\phi \phi}$ ratio is the same for all the components. It is well known that the assumptions of a constant $\sigma_{R R} / \sigma_{\phi \phi}$ ratio, of a linear asymmetric drift and of 2D Gaussian $U$ and $V$ velocity distributions hold only for cold stellar populations (see for instance, Bienaymé \& Séchaud 1997). These simple assumptions allow a direct comparison with similar studies. It allows also an exact integration of count equations along the line of sight. Thus the convergence of parameters for any single model is achieved in a reasonable amount of time (one week). The model includes 20 isothermal components with $\sigma_{z z}$ from 3.5 to $70 \mathrm{~km} \mathrm{~s}^{-1}$. We choose a step of $3.5 \mathrm{~km} \mathrm{~s}^{-1}$ which is sufficient to give a realistic kinematic decomposition and permit calculation in a reasonable time. The two first components $\sigma_{z z}=3.5$ and $7 \mathrm{~km} \mathrm{~s}^{-1}$ were suppressed since they do not contribute significantly to counts for $m_{\mathrm{K}}>6$ and are not constrained by our adjustments. The components between 10 and $60 \mathrm{~km} \mathrm{~s}^{-1}$ are constrained by star counts, proper motions histograms up to magnitude 14 in $K$ and radial velocity histograms for magnitudes $m_{\mathrm{K}}=$ [5.5-11.5]). The model includes isothermal components from 60 to $70 \mathrm{~km} \mathrm{~s}^{-1}$ to properly fit the star counts at the faintest apparent magnitudes $m_{\mathrm{K}}>15.0$. All the values of the kinematic components depend on the adopted galactic potential.

The velocity ellipsoids are inclined along the Galactic meridian plane. The main axis of velocity ellipsoids are set parallel to con-focal hyperboloids as in Stäckel potentials. We set the focus at $z_{\text {hyp }}=6 \mathrm{kpc}$ on the main axis giving them realistic orientations (see Bienaymé 1999). The non-zero inclination implies that the vertical density distributions of each isothermal component is not fully dynamically consistent with the potential. Since the $z$-distances are below $1.5 \mathrm{kpc}$ for the majority of stars with kinematic data, and since the main topic of this paper is not the determination of the Galactic potential, we do not develop a more consistent dynamical model. 


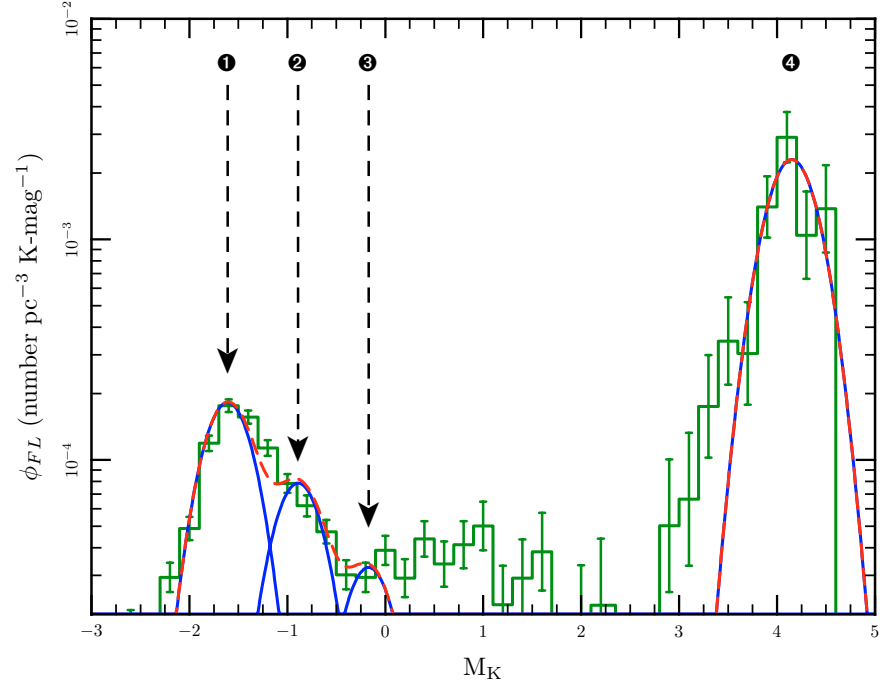

Fig. 3. Local luminosity function: The histogram is our determination of the local luminosity function for nearby stars with error bars. The red (or dark grey) dashed line is a fit of the luminosity function with four gaussians (blue or light grey line) corresponding to the dwarfs, the giants and the two types of sub-giants.

\subsection{The luminosity functions}

The luminosity function of each stellar disk component is modeled with $n$ different kinds of stars according to their absolute magnitude:

$\phi_{i}(M)=\sum_{k=1, n} \phi_{i k}(M)=\frac{1}{\sqrt{2 \pi} \sigma_{M}} \sum_{k=1, n} c_{i k} \mathrm{e}^{-\frac{1}{2}\left(\frac{M-M_{k}}{\sigma_{M}}\right)^{2}}$

where $c_{i k}$ is the density for each type of star (index $k$ ) of each stellar disk component (index $i$ ).

We use four types of stars to model the local luminosity function (see Fig. 3). More details on the way that we have determined it is given in Sect. 4.4. Stars with a mean absolute magnitude $M_{\mathrm{K}}=-1.61$ are identified to be the red clump giants $(k=1)$ that we will call "giants", with $M_{\mathrm{K}}=-0.89$ and $M_{\mathrm{K}}=-0.17$ for first ascent giants that we categorize as "sub-giants" $(k=2-3)$ and $M_{\mathrm{K}}=4.15$ are labelled dwarfs $(k=4)$ (see Fig. 1$)$. We neglected "sub-giant" populations having absolute magnitude $M_{\mathrm{K}}$ between 0.2 and 2 . Their presences marginally change the ratio of giants to dwarfs, since their magnitudes are lower, and their total number in the magnitude counts appears significantly smaller than the other components. In fact, we initially tried to introduce 10 types of stars (spaced by 0.7 absolute magnitude intervals). This still improves the fit to the data. However due to the small contribution of the "sub-giants" components with $M_{\mathrm{K}}=[0.2-2]$, they were not determined with a useful accuracy. We adopt $\sigma_{M}=0.25$, justified by the narrow range of absolute magnitudes both for red clump giants and for dwarfs on the luminosity function.

The $4 \times 20$ coefficients $c_{i k}$ are parameters of the model. In order to obtain a realistic luminosity function, we have added constraints to the minimization procedure. For each kinematic component $i$, we impose conditions on the proportion of dwarfs, giants and sub-giants following the local luminosity function. We have modeled our determination of the local luminosity function of nearby stars (see Fig. 3). We obtained:

- a ratio of the density of dwarfs $(k=4)$ to the density of giants $(k=1)$ of 12.0 , so we impose: $\frac{c_{i, 4}}{c_{i, 1}}>10$
- a ratio of the density of giants $(k=1)$ to the density of subgiants $(k=2)$ of 2.3 , so we impose: $\frac{c_{i, 1}}{c_{i, 2}}>2$

- and the density of sub-giants $(k=2)$ is greater than the density of sub-giants $(k=3)$, so we impose: $c_{i, 1}>c_{i, 2}$.

If we do not include these constraints, the various components are populated either only with dwarfs or only with giants.

\section{Results and discussion}

The 181 free model parameters are adjusted through simulations. Each simulation is compared to histograms of counts, proper motions and radial velocities (see Sect. 2 for the description of data histograms and see Figs. 4-8) for the comparison of the best fit model with data. The adjustment is done by minimizing a $\chi^{2}$ function using the MINUIT software (James 2004). Equal weight is given to each of the four types of data (magnitude counts, $\mu_{U}$ proper motions, $\mu_{V}$ proper motions, and radial velocities). This gives relatively more weight to the radial velocity data whose contribution in number is two orders of magnitude smaller than for the photometry and proper motions.

By adjusting our Galactic model, we derive the respective contributions of dwarfs and giants, and of thin and thick disks. One noticeable result is the kinematic gap between the thin and thick disk components of our Galaxy. This discontinuity must be the consequence of some specific process of formation for these Galactic components.

Fitting a multi-parameter model to a large data-set raises the question of the uniqueness of the best fit model, and the robustness of our solution and conclusions. For this purpose, we have explored the strength of the best Galactic model, by fitting various subsets of data, by modifying various model parameters and adjusting the others. This is a simple, but we expect efficient, way to understand the impact of parameter correlations and to see what is really constrained by model or by data. A summary of the main outcomes is given below.

From these explorations, we choose to fix or bound some important Galactic model parameters which would otherwise be poorly constrained: i) we fix the vertical Galactic potential (adjusting the $K_{z}$ force does not give more accurate results than for instance in Bienaymé et al. 2006, since we only increase by a factor 2 the number of stars with measured radial velocities); ii) the asymmetric drifts of all kinematic components are linked through a unique linear asymmetric drift relation with just one free parameter; the solar velocity component $V_{\odot}$ is also fixed; iii) the axis ratio of the velocity ellipsoids is bounded; for thin disk components $\left(\sigma_{W} \leq 25 \mathrm{~km} \mathrm{~s}^{-1}\right)$ we set $\sigma_{U} / \sigma_{W}>1.5$, for thick $\operatorname{disks}\left(\sigma_{W}>30 \mathrm{~km} \mathrm{~s}^{-1}, \sigma_{U} / \sigma_{W}>1.1\right)$

The agreement between our fitted model and the observed counts is illustrated by the various magnitude, proper motion and radial velocity distributions (Figs. 4-8). We can consider that globally the agreement is good, if we note the small $\chi^{2}$ values obtained. We just comment the main disagreements visible within these distributions. They can be compared to recent similar studies (Girardi et al. 2005, Vallenari et al. 2006).

The agreement for the apparent magnitude distribution looks satisfying in Fig. 4.

The comparison of observed and modeled $\mu_{U}$ proper motion distributions does not show satisfactory agreement close to the maxima of histograms at apparent magnitude $m_{\mathrm{K}}<10$ (NGP or SGP, see Fig. 5). We have not been able to determine if this is due to the inability of our model to describe the observed data, for instance due to simplifying assumptions (gaussianity of the velocity distribution, asymmetric drift relation, constant ratio of 

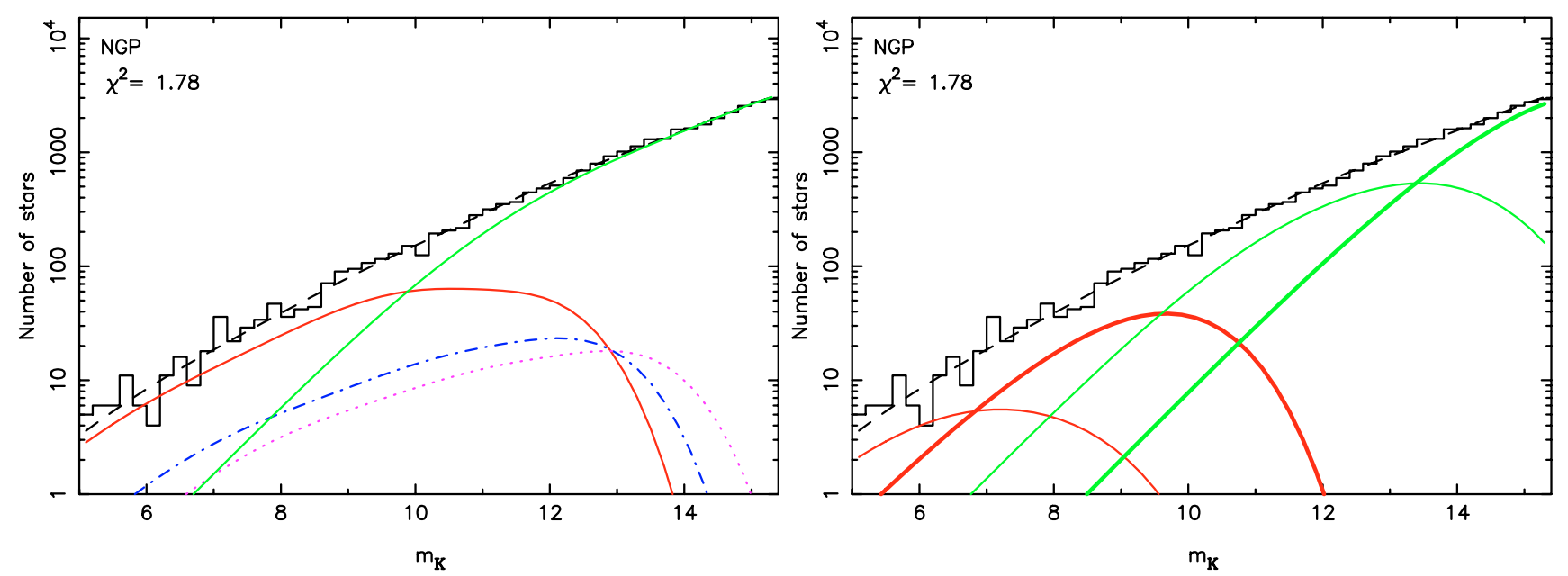

Fig. 4. Magnitude count histogram towards the North Galactic Pole. Left: model prediction (dashed line) is split according to star types: giants (red or black line), sub-giants (dot-dashed and dotted) and dwarfs (green or grey line). The right figure highlights the contributions of thin and thick disks (respectively thin and thick lines), for dwarfs (green or grey) and giants (red or black).
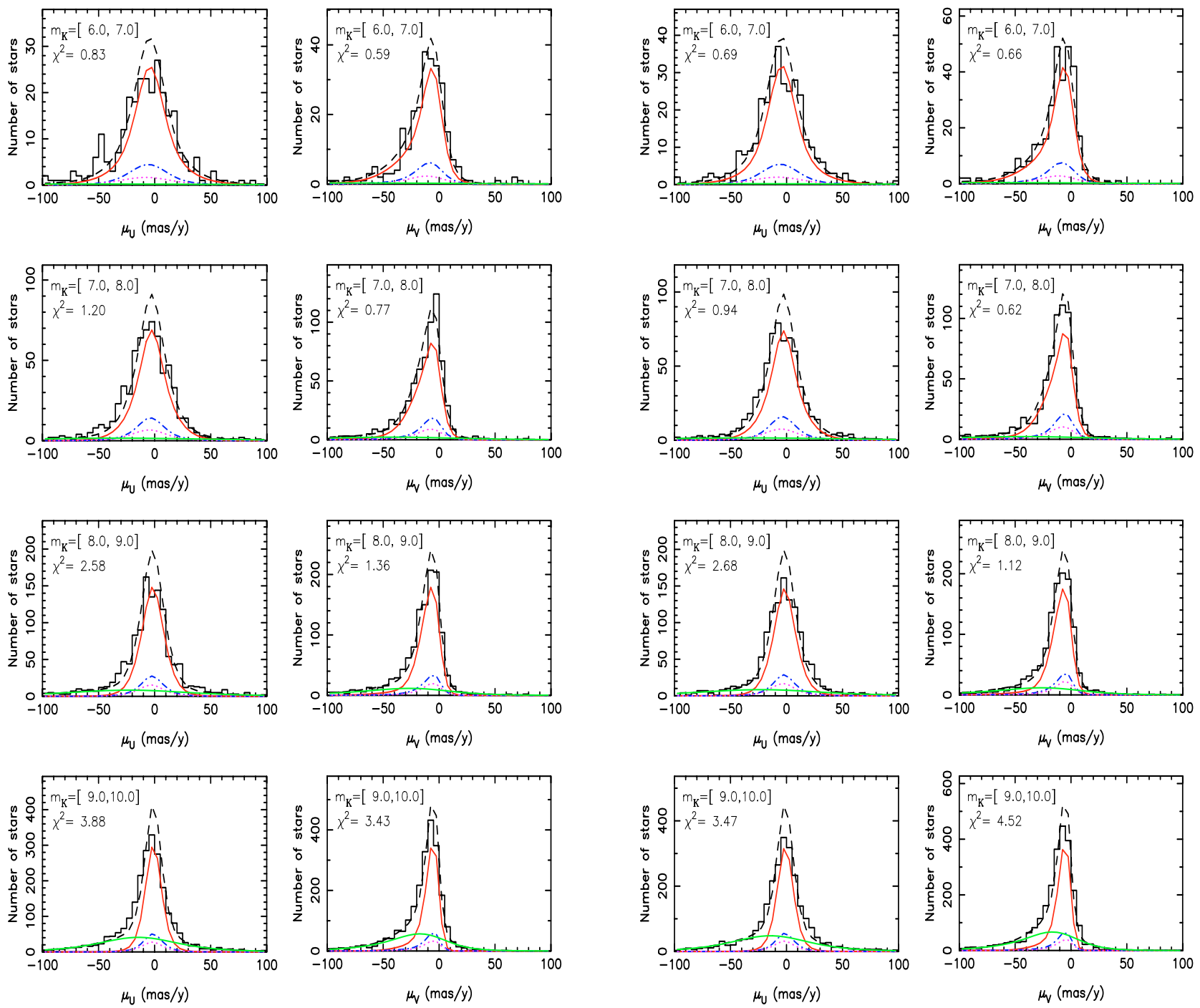

Fig. 5. $\mu_{U}$ and $\mu_{V}$ histograms towards the North Galactic Pole (right) and the South Galactic Pole (left) for magnitudes 6 to 10: model (dashed line) and contributions from the different types of stars: giants (red or dark thin lines), sub-giants (dot-dashed and dotted lines) and dwarfs (green or grey thick lines). 

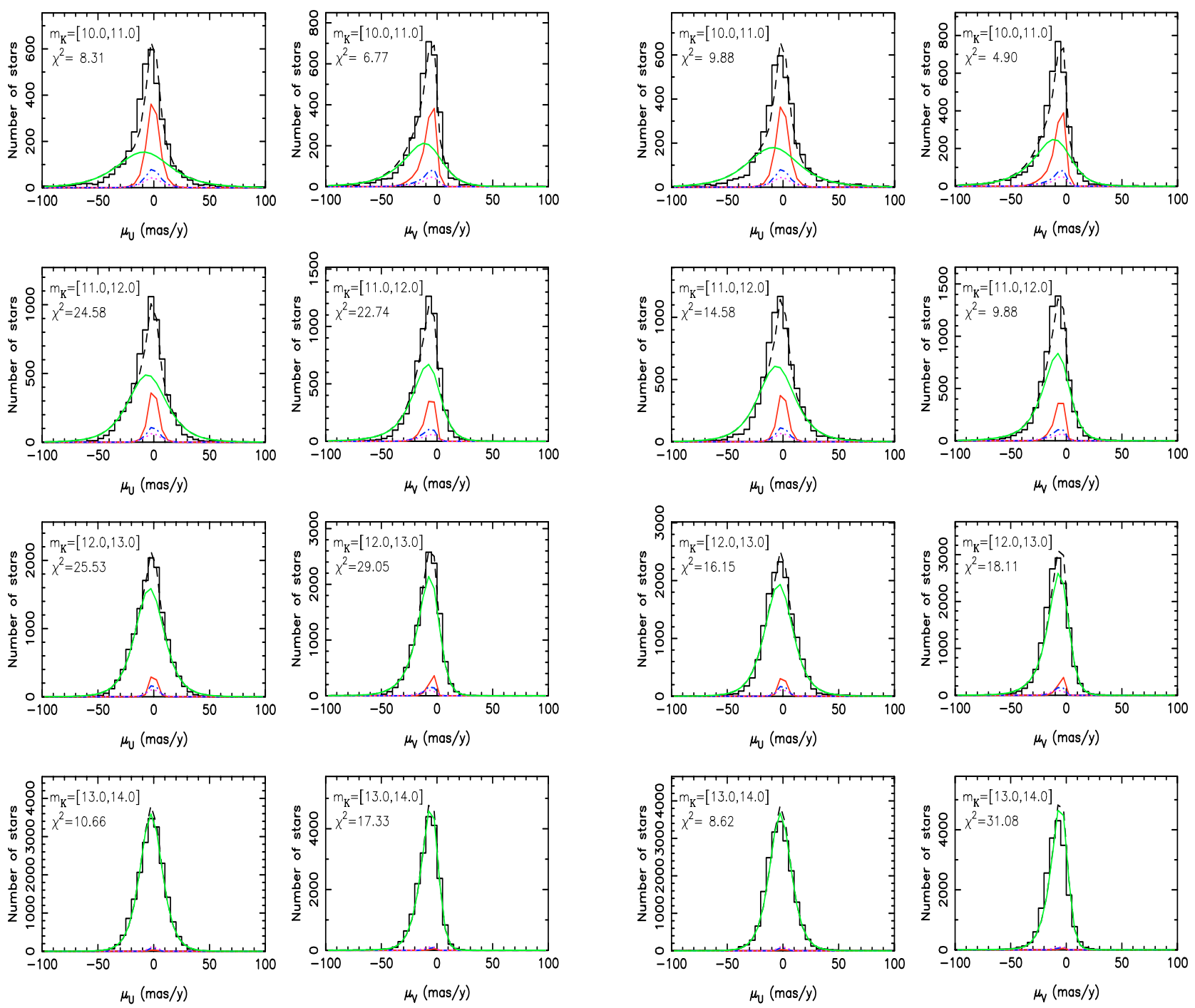

Fig. 6. Same as Fig. 5 for magnitudes 10 to 14 .
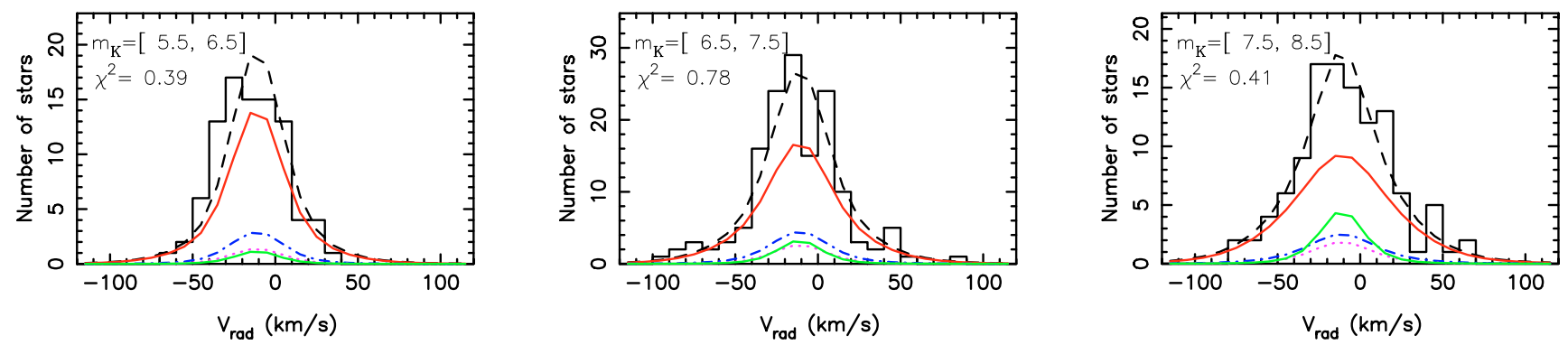

Fig. 7. Radial velocity histograms towards the North Galactic Pole for magnitudes 5.5 to 8.5 for ELODIE data: model (dashed line) and contributions of the different type of stars: giants (red or dark lines), sub-giants (dot-dashed and dotted) and dwarfs (green or grey line).

velocity dispersions, etc.). We note that this disagreement may just result from an underestimate of the impact of the proper motion errors.

Some possible substructures are seen in proper motion histograms for the brightest bins $\left(m_{\mathrm{K}}<7\right.$, Fig. 5); they are close to the level of Poissonian fluctuations and marginally significant. One of the possible structures corresponds to the known
Hercules stream $\left(\bar{U}=-42 \mathrm{~km} \mathrm{~s}^{-1}\right.$ and $\bar{V}=-52 \mathrm{~km} \mathrm{~s}^{-1}$, Famaey et al. 2005).

For faint magnitude $\left(m_{\mathrm{K}}>11\right)$ bins (Fig. 6), small shifts $\left(\sim 3-5 \mathrm{mas} \mathrm{yr}^{-1}\right)$ of $\mu_{U}$ explain most of the differences between North and South and the larger $\chi^{2}$.

At $m_{\mathrm{K}}$ within 10-13 (Fig. 6), the wings of $\mu_{U}$ histograms look slightly different between North and South directions; it 

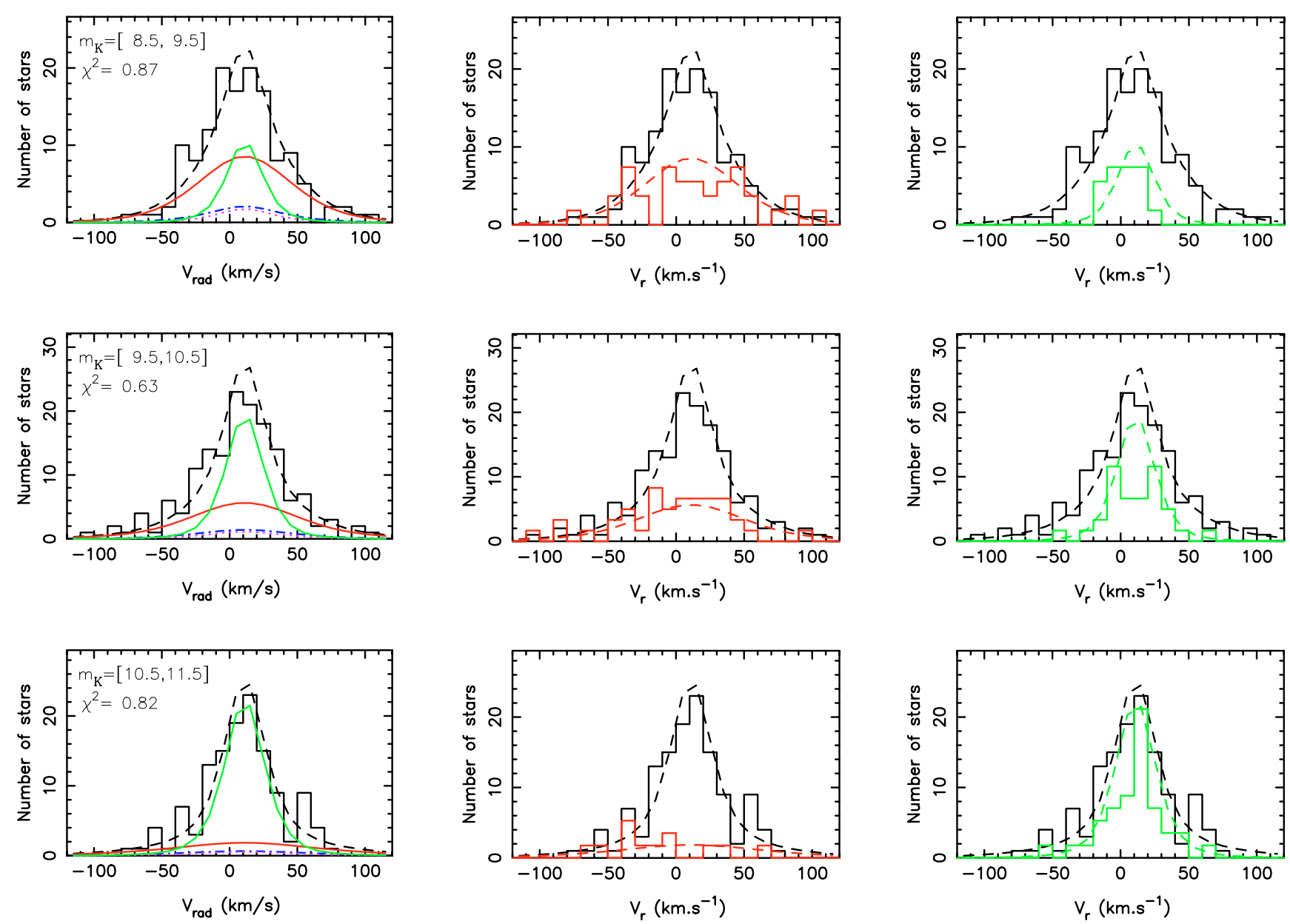

Fig. 8. Number of giants and dwarfs in RAVE data compared to model prediction. Left column: radial velocity histograms towards the South Galactic Pole for magnitudes 8.5 to 11.5 for RAVE data, model (dashed line) and contributions of the different type of stars: giants (red or dark lines), sub-giants (dot-dashed and dotted lines) and dwarfs (green or grey line). Center column: radial velocity histograms for all stars (black) and for giants (red or grey): model for all stars (black dashed line) and for giants (red or grey dashed line). Right column: radial velocity histograms for all stars (black) and for dwarfs (green or light grey): model for all stars (black dashed line) and for dwarfs (green or light grey dashed line).

apparently results from shifts of North histograms versus South ones.

A disagreement of the model versus observations also appears within the wings of $\mu_{V}$ distributions, ( $m_{\mathrm{K}}$ within 10-13, Fig. 6). This may introduce some doubt concerning our ability to correctly recover the asymmetric drift, because the negative proper motion tail of $\mu_{V}$ distributions directly reflects the asymmetric drift of the $V$ velocity component. However, we estimate that our determination of the asymmetric drift coefficient is robust and marginally correlated to the other model parameters.

These comparisons of observed and model distributions suggest new directions to analyze data. In the future, we plan to use the present galactic model to simultaneously fit the RAVE radial velocity distribution in all available galactic directions. This result will be compared to a fit of our model to proper motion distributions over all galactic directions. This will give a better insight into the inconsistency between radial velocity and proper motion data, and also for possible inconsistency in our galactic modeling.

\subsection{The transition from dwarfs to giants}

Within the $J-K=[0.5-0.7]$ interval, the proper motion is an excellent distance indicator: there is a factor of 14 between the proper motion of a dwarf and the proper motion of a giant with the same apparent magnitudes and velocities. Combining proper motions and apparent magnitudes, our best-fit Galactic model allows us to separate the contributions of dwarfs and giants (Fig. 4).

We deduce that, towards the Galactic poles, most of the bright stars are giants. At $m_{\mathrm{K}}=7.2$, only $10 \%$ are dwarfs and at $m_{\mathrm{K}}=9.6$ only $50 \%$ are giants. We have checked if the contribution of sub-giants with absolute magnitude $M_{\mathrm{K}}=[0.2-2]$ can change the contribution of dwarfs and giants. At $m_{\mathrm{K}}<10$, the contribution of sub-gaint with $M_{\mathrm{K}}=[0.2-2]$ is at least one order of magnitude lower. So the ratio of giants and dwarfs is unchanged. Furthermore, the RAVE data confirm our model prediction. This is in contradiction with Cabrera-Lavers et al. (2005) statement based on the Wainscoat et al. (1992) model which estimates that, at magnitude $m_{\mathrm{K}}<10$, giants represent more than $90 \%$ of the stars. The Wainscoat model assumes only one disk with a scale height of $270 \mathrm{pc}$ for the giants and $325 \mathrm{pc}$ for the dwarfs. In our model, we find a scale height of $225 \mathrm{pc}$ both for the giants and the dwarfs. This explains why we find more dwarfs at bright magnitudes $\left(m_{\mathrm{K}}<10\right)$.

Faint stars are mainly dwarfs, $80 \%$ at $m_{\mathrm{K}}=11.6$ while at $m_{\mathrm{K}}=11.9$, only $10 \%$ are giants. The $50 \%-50 \%$ transition between giants-sub-giants and dwarfs occurs at $m_{\mathrm{K}} \sim 10.1$. This is 


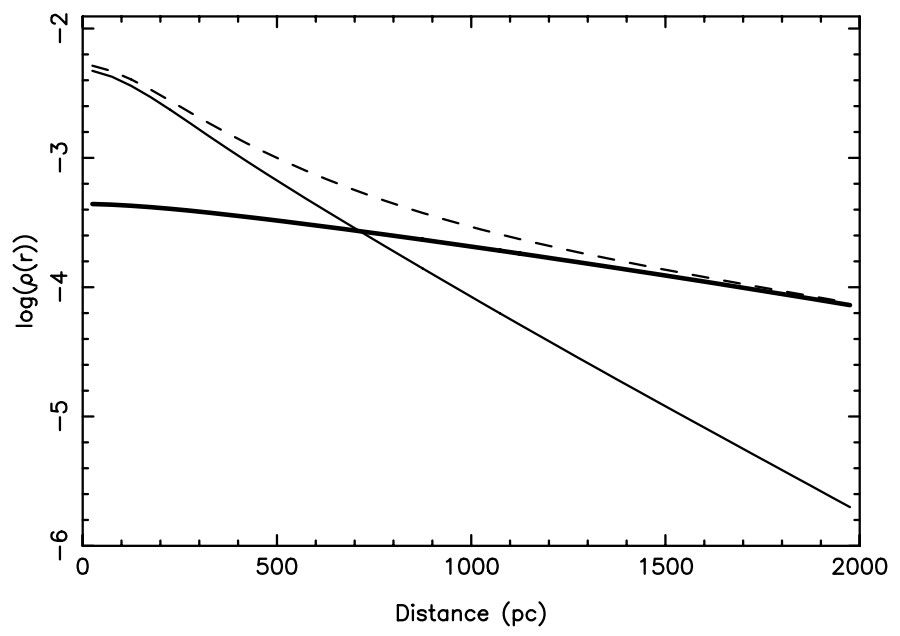

Fig. 9. Model of the vertical stellar density $\rho(z)$ towards the the North Galactic Pole (dashed line) and its thin and thick disk decomposition (respectively thin and thick lines). The thin disk includes the isothermal kinematic components with $\sigma_{W}<25 \mathrm{~km} \mathrm{~s}^{-1}$, the thick disks include components with $\sigma_{W}>25 \mathrm{~km} \mathrm{~s}^{-1}$.

Table 1. List of the values of the kinematic disk components $\phi_{\mathrm{kin}, i}\left(10^{6} \times\right.$ number of stars $/ \mathrm{pc}^{3}$ ) with the individual errors absolutes and relatives in percent.

\begin{tabular}{rcrcc}
\hline \hline No. & $\begin{array}{c}\sigma_{w} \\
\left(\mathrm{~km} \mathrm{~s}^{-1}\right)\end{array}$ & $\begin{array}{c}\phi_{\text {kin }} \\
\left(\times 10^{6}\right)\end{array}$ & $\begin{array}{c}\text { Error } \\
\text { absolute }\end{array}$ & $\begin{array}{c}\text { Error } \\
\text { in } \%\end{array}$ \\
\hline 1 & 3.5 & 0.00 & - & - \\
2 & 7.0 & 0.00 & - & - \\
3 & 10.5 & 2044.13 & 720.50 & 35.25 \\
4 & 14.0 & 596.69 & 493.81 & 82.76 \\
5 & 17.5 & 1618.79 & 169.57 & 10.48 \\
6 & 21.0 & 385.76 & 92.03 & 23.86 \\
7 & 24.5 & 234.53 & 54.72 & 23.33 \\
8 & 28.0 & 3.85 & 35.10 & $>100$ \\
9 & 31.5 & 53.21 & 33.09 & 62.19 \\
10 & 35.0 & 79.16 & 30.73 & 38.82 \\
11 & 38.5 & 64.71 & 63.76 & 98.53 \\
12 & 42.0 & 27.49 & 66.31 & $>100$ \\
13 & 45.5 & 216.96 & 44.07 & 20.32 \\
14 & 49.0 & 2.63 & 39.19 & $>100$ \\
15 & 52.5 & 0.38 & 0.08 & 21.05 \\
16 & 56.0 & 0.04 & 0.04 & 100.00 \\
17 & 59.5 & 0.29 & 0.11 & 37.93 \\
18 & 63.0 & 4.83 & 31.72 & $>100$ \\
19 & 66.5 & 5.86 & 30.88 & $>100$ \\
20 & 70.0 & 2.69 & 0.05 & 1.86 \\
\hline
\end{tabular}

a robust result from our study that depends slightly on the absolute magnitude adopted for dwarf and giant stars. We have not tried to change our color range. If we take a broader color interval, the dispersion around the absolute magnitude of dwarfs will be larger, but our results are not expected to change. For another color interval, we can expect this result to be different, since we would be looking at a different spectral type of star.

A confirmation of the dwarf-giant separation between magnitudes $m_{\mathrm{K}}=$ [5.5-11.5] comes from RAVE spectra. With the preliminary determination of the stellar parameters $\left(T_{\text {eff }}, \log (g)\right.$ and $[\mathrm{Fe} / \mathrm{H}]$ ) of RAVE stars, we choose to define giant stars with $\log (g)<3$ and dwarfs with $\log (g)>4$. The comparison of the number of giants and dwarfs predicted by our best model to the observed one is in good agreement (see Fig. 8).

\subsection{The scale heights of stellar components}

Our dynamical modeling of star counts allows us to recover the vertical density distribution of each kinematic component $\rho_{i}(z)$, with the exact shapes depending on the adopted vertical potential $\Phi(z)$. We recover the well-known double-exponential shape of the total vertical number density distribution $\rho_{\text {tot }}(z)$ (Fig. 9). Since we estimate that the kinematic decomposition in isothermal components is closer to the idealized concept of stellar populations and disks, we identify the thin disk as the components with vertical velocity dispersions $\sigma_{W}$ smaller than $25 \mathrm{~km} \mathrm{~s}^{-1}$ and the thick disk with $\sigma_{W}$ from 30 to $45.5 \mathrm{~km} \mathrm{~s}^{-1}$ (Fig. 12). Following this identification, we can fit an exponential on the thin and thick disk vertical density component (thin line and thick lines respectively of Fig. 9). The scale height of the thin disk is $225 \pm 10$ pc within $200-800$ pc. For the thick disk, within $0.2-1.5 \mathrm{kpc}$, the scale height is $1048 \pm 36 \mathrm{pc}$. If we consider all the kinematic components without distinguishing between the thin and thick disk, we can fit a double exponential with a scale length of the thin disk $217 \pm 15 \mathrm{pc}$ and of the thick disk $1064 \pm 38$ pc. We calculate the error of the scale length from the error on the individual kinematic disk components $\phi_{\mathrm{kin}, i}$ (see Table 1). We have performed a Monte-Carlo simulation on the value of the components and obtained the error bars for the scale length of the thin and thick disk both independently and together.

We note that our density distribution is not exponential for $z<200$ pc: this mainly results from the fact that we do not model components with small velocity dispersions $\sigma_{W}<8 \mathrm{~km} \mathrm{~s}^{-1}$. Thus our estimated density at $z=0$ cannot be directly compared, for instance, to Cabrera-Lavers et al. (2005) results. With this proviso, the star number density ratio of thick to thin disk stars at $z=0 \mathrm{pc}$ is $8.7 \%$ for the dwarfs.

One candidate to trace the thin and thick disk are the red clump giants. In fact, at $z$-distances larger than $\sim 500 \mathrm{pc}$ (i.e. $m_{\mathrm{K}}$ larger than $\sim 7.0$, see Fig. 4, there are more thick disk giants than thin disk giants. Cabrera-Lavers et al. (2005) have analyzed them using 2MASS data. To do this, they select all stars with color $J-K=[0.5-0.7]$ and magnitude $m_{\mathrm{K}}<10$. But, beyond magnitude 9 , the proportion of giants relative to sub-giants and dwarfs decreases quickly. At $m_{\mathrm{K}}=9.6$, giants just represent half of the stars, and their distance is about $1.7 \mathrm{kpc}$. Thus, we must be cautious when probing the thick disk with clump giants and we have first to determine the respective sub-giant and dwarf contributions. However, Cabrera-Lavers et al. (2005) obtained a scale height of $267 \pm 13 \mathrm{pc}$ and $1062 \pm 52 \mathrm{pc}$ for the thin and thick disks which is in relatively good agreement with the values obtained from our model.

For dwarfs that dominate the counts at faint apparent magnitudes $m_{\mathrm{K}}>11$ (distances larger than $\sim 240 \mathrm{pc}$ ), we use the photometric distance:

$z_{\text {phot }}=10^{\left(m_{\mathrm{K}}-M_{\mathrm{K}}-5\right) / 5}$

where $M_{\mathrm{K}}$ is equal to 4.15 (the value for the dwarfs).

Doing so, we obtain the number density $n\left(z_{\text {phot }}\right)$ of stars seen along the line of sight at the SGP and NGP (Fig. 10). These plots show a well-defined first maximum at $z_{\text {phot }}=500 \mathrm{pc}$ (SGP) or $700 \mathrm{pc}$ (NGP) related to the distribution of thin disk dwarfs. At $0.9-1.1 \mathrm{kpc}, n\left(z_{\text {phot }}\right)$ has a minimum and then rises again at larger distances, indicating the thick disk dwarf contribution.

However, the use of photometric distances can introduce a systematic error for thick disk dwarfs that have lower metallicities. The mean metallicity of the thick disk population at $1 \mathrm{kpc}$ is $\langle[\mathrm{Fe} / \mathrm{H}]\rangle \simeq-0.6$ (Gilmore et al. 1995; Carraro et al. 1998; Soubiran et al. 2003). 

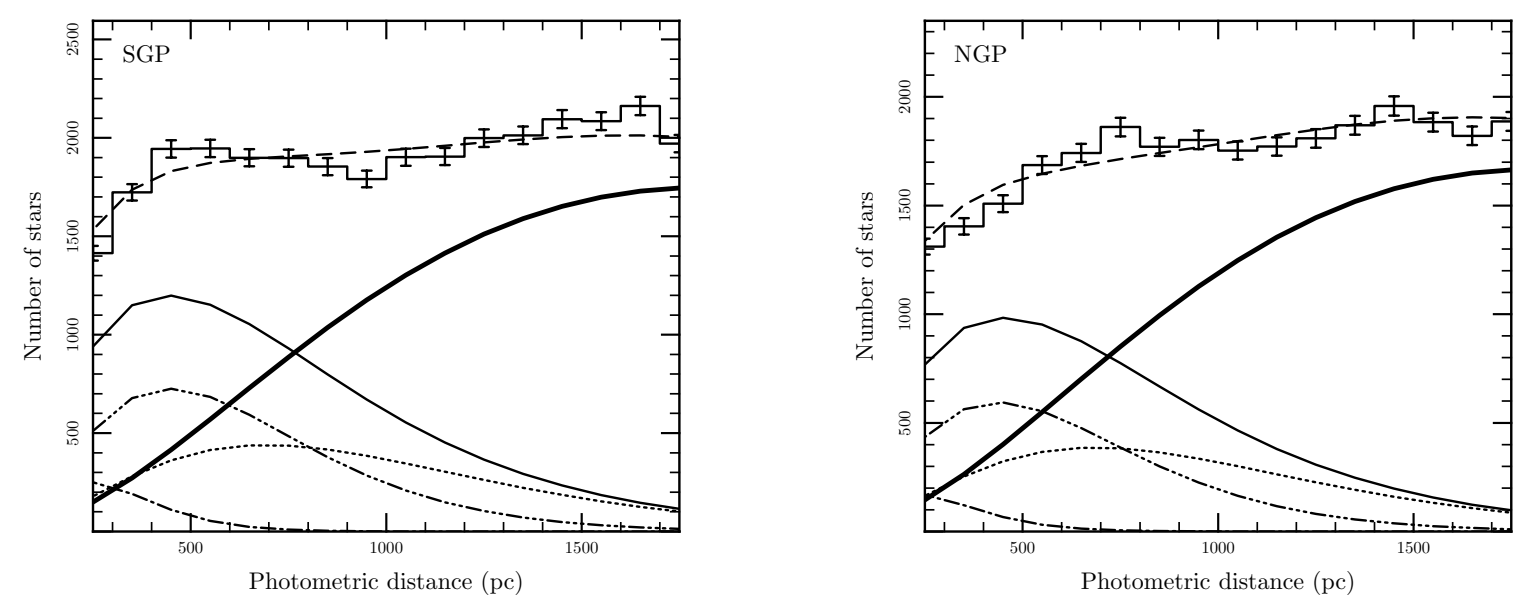

Fig. 10. Data (histogram with error bars) and model (dashed line) for the NGP (left) and SGP (right) vertical density distribution using photometric distances $n_{\text {phot }}(z)$ for dwarf stars. The transition between thin and thick components is revealed by a minimum at $z \sim 1 \mathrm{kpc}$. The main contributing components are plotted, for the thin disk (thin continuous line) $\sigma_{W}=10.5$ (dot-dashed), 14 and 17.5 (triple dot-dashed), 21 and $24.5 \mathrm{~km} \mathrm{~s}{ }^{-1}$ (dotted) and for the thick disk (thick continuous line) $\sigma_{W}=45.5 \mathrm{~km} \mathrm{~s}^{-1}$.

The metallicity variation from $[\mathrm{Fe} / \mathrm{H}]=0.0$ for the thin disk to $[\mathrm{Fe} / \mathrm{H}]=-0.6$ for the thick disk means that the absolute magnitude $M_{\mathrm{K}}$ changes from 4.15 to 4.5 . So, we smoothly vary the absolute magnitude with the metallicity from the thin to the thick disk, in this way:

$M_{\mathrm{K}}([\mathrm{Fe} / \mathrm{H}])=M_{\mathrm{K}, 0}+0.035 m_{\mathrm{K}}$

where $M_{\mathrm{K}, 0}$ is equal to 4.15 .

The counts continue to show two maxima (Fig. 11), even if the minimum is less deep. The minimum delineates a discontinuous transition between the thin and thick components.

The superposition of the model on the number density $n\left(z_{\text {phot }}\right)$ shows only approximate agreement (Fig. 10). We think that is due to non-isothermality of the real stellar components. Anyway, the fact that the model does not reproduce exactly the observation does not weaken the conclusion about the kinematic separation of the thin and thick disk. It reinforces the need for a clear kinematic separation between the two disks in the kinematic decomposition (Fig. 12).

We also notice, in Fig. 10, the difference in counts between the North and the South. This difference allows us to determine the distance of the Sun above the Galactic plane, $z_{\odot}=$ $+20.0 \pm 2.0 \mathrm{pc}$, assuming symmetry between North and South. We also note that the transition between thin and thick disks is more visible towards the SGP than towards the NGP.

\subsection{The thin-thick disk transition, and the kinematic distribution function}

The minimum at $z \sim 1 \mathrm{kpc}$ in the $n(z)$ distribution (Fig. 10) provides very direct evidence of the discontinuity between stellar components with small velocity dispersions $\left(\sigma_{W}=10-25 \mathrm{~km} \mathrm{~s}^{-1}\right)$ and those with intermediate velocity dispersions $\left(\sigma_{W} \sim 45.5 \mathrm{~km} \mathrm{~s}^{-1}\right)$ (left panel Fig. 12).

Another manifestation of this transition is well known from the $\log \rho(z)$ density distribution (Fig. 9) which shows a change of slope at $z=500-700 \mathrm{pc}$. This feature can be successfully modeled with two (thin and thick) components (e.g. Reid \& Gilmore 1983), which is an indication of a discontinuity between the thin and thick disks of our Galaxy.

It is conclusive evidence, only if we show that we can not fit accurately the star counts or vertical density distributions with a continuous set of kinematic components (without a gap between the thin and the thick disks). We find that the constraint of a set of kinematic components following a continuous trend (right panel of Fig. 12) raises the reduced $\chi^{2}$, in particular on SGP magnitude counts, from 1.59 to 3.40. This confirms the robustness of our result and conclusion on the wide transition between thin and thick stellar disk components.

Adjusting the Galactic model to star counts, tangential and radial velocities, we can recover the details of the kinematics of stellar populations, and we determine the local $\sigma_{W}$ kinematic distribution function (left panel of Fig. 12 and Table 1). This kinematic distribution function clearly shows a large step between the kinematic properties of the thin and thick disks. We define the thin disk as the components with $\sigma_{W}$ covering $10-25 \mathrm{~km} \mathrm{~s}^{-1}$, and the thick disk as the components with $\sigma_{W}$ covering $30-45 \mathrm{~km} \mathrm{~s}^{-1}$. The counts and radial velocities by themselves already show the kinematic transition that we obtain in the kinematic decomposition. The fit of proper motions confirms the conclusion from the star counts and radial velocities, even if a fraction of the proper motions $\mu_{l}$ and $\mu_{b}$ at magnitude $m_{\mathrm{K}}$ fainter than 13 have significant errors $\left(>20 \mathrm{~km} \mathrm{~s}^{-1}\right)$. The only consequence for the proper motion errors is that we obtained an ellipsoid axis ratio $\sigma_{U} / \sigma_{W}$ different from the classical values (see Sect. 4.5).

The last non-null components at approximately $\sigma_{W} \sim$ $65 \mathrm{~km} \mathrm{~s}^{-1}$ are necessary to fit the faintest star counts at $m_{\mathrm{K}} \sim 15$. But, they do not result from the fit of proper motion histograms (since, unfortunately, they stop at $m_{\mathrm{K}} \sim 14$ ). Thus their exact nature, a second thick disk or halo (they would have very different asymmetric drift) cannot be solved in the context of our analysis.

\subsection{The luminosity function of stellar components}

Our distant star count and kinematic adjustment constrains the local luminosity function (LF). We make the comparison with the local LF determined with nearby stars. However, the brightest HIPPARCOS stars needed to determine the local LF are saturated within 2MASS and have less accurate photometry. We can also compare it to the LF determined by Cabrera-Lavers et al. (2005) who use a cross-match of HIPPARCOS and MSX stars and estimate $m_{\mathrm{K}}$ magnitudes from MSX A band magnitudes 

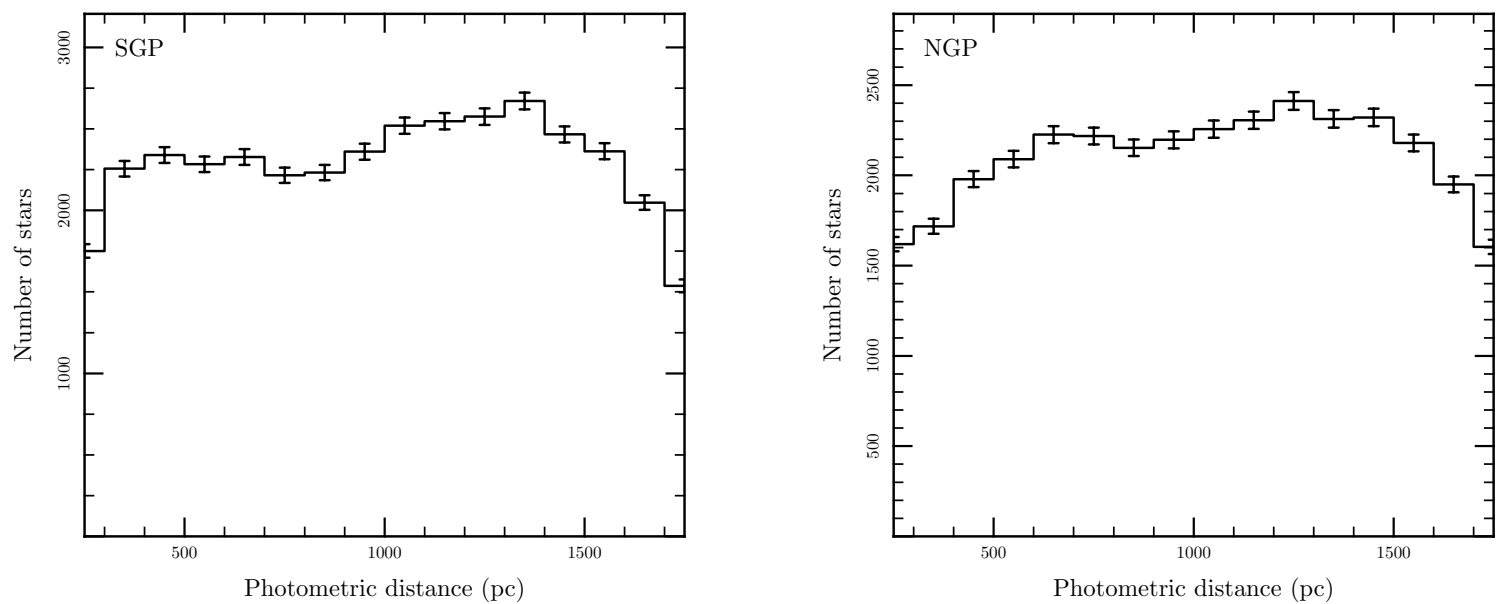

Fig. 11. Histograms of the vertical density distribution for the NGP (left) and SGP (right) using photometric distances $n_{\text {phot }}(z)$ for dwarf stars with a smooth variation in the $[\mathrm{Fe} / \mathrm{H}]$ from the thin to the thick disk.
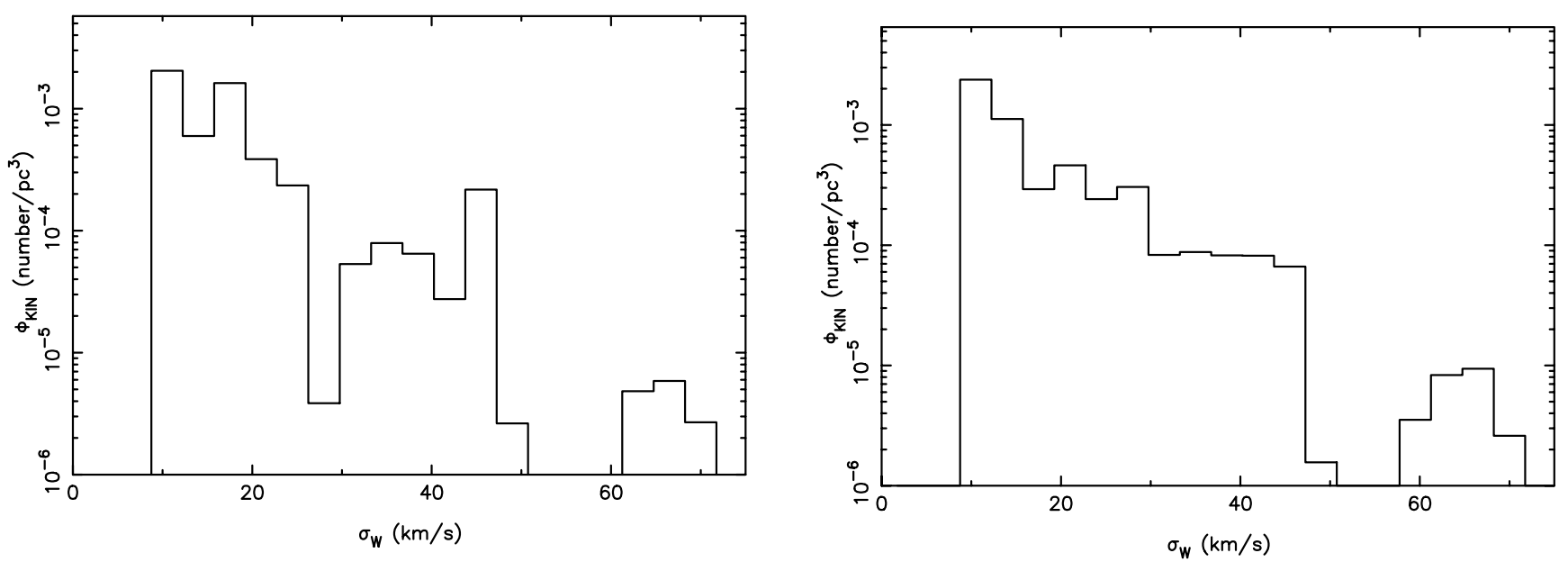

Fig. 12. Left: the local $\sigma_{W}$ kinematic distribution function. The contributing components to star counts can be put together in a thin disk component $\left(\sigma_{W}<25 \mathrm{~km} \mathrm{~s}^{-1}\right)$, a thick disk (isothermal with $\left.\sigma_{W}=45.5 \mathrm{~km} \mathrm{~s}^{-1}\right)$ and a hotter component with $\sigma_{W} \sim 65 \mathrm{~km} \mathrm{~s}^{-1}$. The two first components with $\sigma_{W}=3.5$ and $7 \mathrm{~km} \mathrm{~s}^{-1}$ are set to zero by construction. Right: a Kinematic Distribution Function (KDF) that tries to reproduce the magnitude star counts and the kinematic data: this model has been obtained requiring the continuity of the KDF from $\sigma_{w}=10$ to $48 \mathrm{~km} \mathrm{~s}^{-1}$.

(hereafter [8.3]). However we note from our own cross-match of HIPPARCOS-MSX-2MASS (non saturated) stars that their LF, for stars selected from V-[8.3], corresponds mainly to stars with $J-K$ colors between $0.6-0.7$ rather than between $0.5-0.7$. A second limitation for a comparison of LFs is that our modeling does not include the stellar populations with small velocity dispersions $\left(\sigma_{W}<8 \mathrm{~km} \mathrm{~s}^{-1}\right)$. For these reasons, we determine a rough local LF based on 2MASS-HIPPARCOS cross-matches, keeping stars with $V<7.3$ or distances $<125 \mathrm{pc}$, and using the color selection $V-K$ between 2.0 and 2.6, that corresponds approximately to $J-K=[0.5-0.7]$. Using $V$ and $K$ mag minimizes the effects of the $J-K$ uncertainties. Considering these limitations, there is reasonable agreement between the local LF obtained with our model using distant stars and the LF obtained from nearby Hipparcos stars (see Fig. 13).

\subsection{The stellar kinematics}

Many of the stellar disk kinematic properties obtained with our best fit Galactic model are comparable with previously published results. We make the comparison with the analysis of HIPPARCOS data (Dehnen \& Binney 1998; Bienaymé 1999;

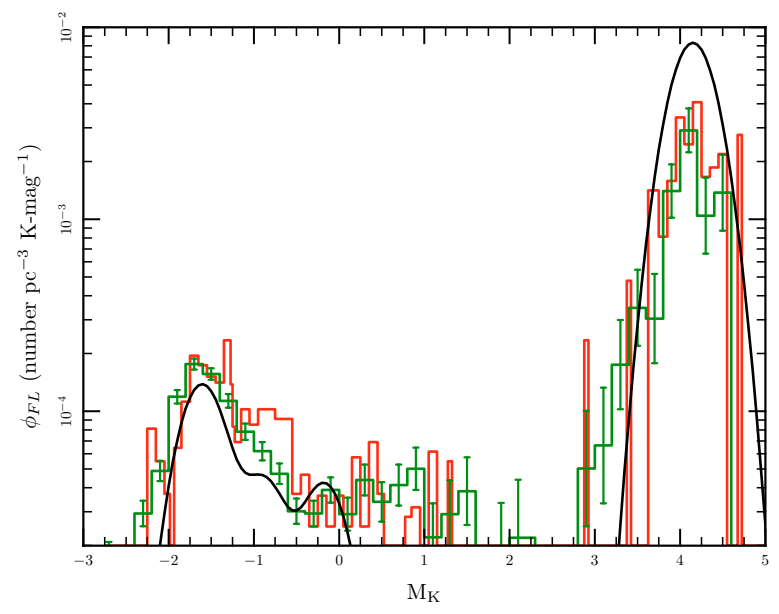

Fig. 13. The local luminosity function of $\mathrm{K}$ stars from our modeling of star counts towards the Galactic poles (line) compared to the LF function from nearby Hipparcos K stars by Cabrera-Lavers et al. (2005) (red or black histogram) and our own estimate of the local LF: see text (green or grey histogram with error bars). The scale of Cabrera et al.'s LF has been arbitrarily shifted. 
Nördstrom et al. 2004; Cubarsi \& Alcobé 2004; Famaey et al. 2005), and also with results published from remote stellar samples using a wide variety of processes to identify thin and thick kinematic components (Bartašiūtė 1994; Flynn \& Morrel 1997; Soubiran et al. 2003; Pauli et al. 2005).

We obtain for the Sun motion relative to the LSR, $u_{\odot}=$ $8.5 \pm 0.3 \mathrm{~km} \mathrm{~s}^{-1}$ and $w_{\odot}=11.1 \pm 1.0 \mathrm{~km} \mathrm{~s}^{-1}$. We find for the asymmetric drift coefficient, $k_{a}=76 \pm 4 \mathrm{~km} \mathrm{~s}^{-1}$, compared to $80 \pm 5 \mathrm{~km} \mathrm{~s}^{-1}$ for nearby HIPPARCOS stars (Dehnen \& Binney 1998) and the thick disk lag is $V_{\text {lag }}=\sigma_{R}^{2} / k_{a}=33 \pm 2 \mathrm{~km} \mathrm{~s}^{-1}$ relative to the LSR. We note that this value of the thick disk lag is close to the value of Chiba \& Beers (2000) and other estimates prior to this. It is less in agreement with the often-mentioned values of $50-100 \mathrm{~km} \mathrm{~s}^{-1}$ from pencil-beam samples. These may be more affected by Arcturus group stars which are more dominant at higher $z$-values.

Our determination of the asymmetric drift coefficient is highly correlated to $V_{\odot}$. The reason is that we do not fit populations with low velocity dispersions and small $V_{\text {lag }}$ since we do not fit star counts with $m_{\mathrm{K}}<6$ : as a consequence the slope of the relation, $V_{\text {lag }}$ versus $\sigma_{U}$, is less well constrained. To improve the $k_{a}$ determination, we adopt $V_{\odot}=5.2 \mathrm{~km} \mathrm{~s}^{-1}$ (Dehnen \& Binney 1998; Bienaymé 1999). The adjusted $\sigma_{U} / \sigma_{V}$ velocity dispersion ratio, taken to be the same for all components, is $1.44 \pm 0.02$. We obtain $\sigma_{U} / \sigma_{W}$ ratios significantly smaller than those published using nearby samples of stars. For the thin disk components, we find $\sigma_{U} / \sigma_{W}=1.50$ to 1.62 (compared to published values $\sim 2$ by authors using HIPPARCOS stars). For the thick disk, we obtain $\sigma_{U} / \sigma_{W}=1.1$, instead of $\sim 1.5-1.7$ typically obtained with nearby thick disk stars by other authors.

While there is no dynamical reason preventing the variation of $\sigma_{U} / \sigma_{W}$ with $z$, we suspect that our low $\sigma_{U} / \sigma_{W}$ ratio at large $z$ for the thick disk results from a bias within our model due to the outer part of the wings of some proper motion histograms not being accurately adjusted. This may be the consequence of an incorrect adopted vertical potential or, as we think, more likely the non-isothermality of the real velocity distributions. This suspicion is reinforced since fitting each proper motion histograms separately with a set of Gaussians gives us larger values for $\sigma_{U} / \sigma_{W}$.

Our results can be directly compared with the very recent analysis by Vallenari et al. (2006) of stellar populations towards the NGP using BVR photometry and proper motions (Spagna et al. 1996). Their model is dynamically consistent but based on quite different hypotheses from ours; for each stellar population, they assume that in the Galactic plane $\sigma_{z z}^{2}$ is proportional to the stellar density $\rho$ (Kruit \& Searle 1982). They also assume that both velocity dispersions, $\sigma_{z z}^{2}$ and $\sigma_{R R}^{2}$, follow exponential laws with the same scale exponential profile as the surface mass density (Lewis \& Freeman 1989). Vallenari et al. (2006) found thick disk properties (see their Table 6) quite similar to the ones obtained in this paper. They obtain: $\sigma_{W}=38 \pm 7 \mathrm{~km} \mathrm{~s}^{-1}$, $\sigma_{U} / \sigma_{V}=1.48, V_{\text {lag }}=42 \pm 7 \mathrm{~km} \mathrm{~s}^{-1}$, and for the thick disk scale height: $900 \mathrm{pc}$. However, they find $\sigma_{U} / \sigma_{W}=1.9$. They also claim that "no significant velocity gradient is found in the thick disk", implying that the thick disk must be an isothermal component.

\subsubsection{Radial velocities}

The number of RAVE and ELODIE stars used in this analysis is a tiny fraction of the total number of stars used from 2MASS or UCAC2 catalogues. However they play a key role in constraining Galactic model parameters: the magnitude coverage of RAVE stars towards the SGP, from $m_{\mathrm{K}}=8.5$ to 11.5 , can be used to discriminate between the respective contributions from each type of star, dwarfs, sub-giants, giants. A future RAVE data release (Zwitter et al. submitted) will include gravities, allowing for easier identification of dwarfs and red clump giants; it will also include element abundances allowing for better description of stellar disk populations and new insights into the process of their formation.

\section{Conclusion}

We revisit the thin-thick disk transition using star counts and kinematic data towards the Galactic poles. Our Galactic modeling of star count, proper motion and radial velocity allows us to recover the LF, their kinematic distribution function, their vertical density distribution, the relative distribution of giants, subgiants and dwarfs, the relative contribution from thin and thick disk components, the asymmetric drift coefficient and the solar velocity relative to the LSR.

The double exponential fitting of the vertical disk stellar density distribution is not sufficient to fully characterize the thin and thick disks. A more complete description of the stellar disk is given by its kinematical decomposition.

From the star counts, we see a sharp transition between the thick and thin components. Combining star counts with kinematic data, and applying a model with 20 kinematic components, we discover a gap between the vertical velocity dispersions of thin disk components with $\sigma_{W}$ less than $21 \mathrm{~km} \mathrm{~s}^{-1}$ and a dominant thick disk component at $\sigma_{W}=45.5 \mathrm{~km} \mathrm{~s}^{-1}$. The thick disk scale height is found to be $1048 \pm 36 \mathrm{pc}$. We identify this thick disk with the intermediate metallicity $([\mathrm{Fe} / \mathrm{H}] \sim-0.6$ to -0.25$)$ thick disk described, for instance, by Soubiran et al. (2003). This thick disk is also similar to the thick disk measured by Vallenari et al. (2006) who find "no significant velocity gradient" for this stellar component. We note that star counts at $m_{\mathrm{K}} \sim 15$ suggest a second thick disk or halo component with $\sigma_{W} \sim 65 \mathrm{~km} \mathrm{~s}^{-1}$.

Due to the separation of the thin and thick components, clearly identified with stars counts and visible within the kinematics, the thick disk measured in this paper cannot be the result of dynamical heating of the thin disk by massive molecular clouds or by spiral arms. We would expect otherwise a continuous kinematic distribution function with significant kinematic components covering without discontinuity the range of $\sigma_{W}$ from 10 to $45 \mathrm{~km} \mathrm{~s}^{-1}$.

We find that, at the solar position, the surface mass density of the thick disk is $27 \%$ of the surface mass density of the thin disk. The thick disk has velocity dispersions $\sigma_{U}=50 \mathrm{~km} \mathrm{~s}^{-1}$, $\sigma_{W}=45.5 \mathrm{~km} \mathrm{~s}^{-1}$, and asymmetric drift $V_{\mathrm{lag}}=33 \pm 2 \mathrm{~km} \mathrm{~s}^{-1}$. Although clearly separated from the thin disk, this thick component remains a relatively "cold" thick disk and has characteristics that are close to the thin disk properties. This "cold" and rapidly rotating thick disk is similar to the component identified by many kinematic studies of the thick disk (see Chiba \& Beers 2000, for a summary). Its kinematics appear to be different from the thick disk stars studied at intermediate latitudes in pencil beam surveys (eg Gilmore et al. 2002), which appear to be significantly affected by a substantial stellar stream with a large lag velocity. They interpret this stellar stream as the possible debris of an accreted satellite (Gilmore 2002; Wyse et al. 2006). Maybe some connections exist with streams identified in the solar neighborhood as the Arcturus stream (Navarro et al. 2004).

Some mechanisms of formation connecting a thin and a thick components are compatible with our findings. It may be, for 
instance a "puffed-up" thick disk, i.e. an earlier thin disk puffed up by the accretion of a satellite (Quinn et al. 1993). Another possibility, within the monolithic collapse scenario, is a thick disk formed from gas with a large vertical scale height before the final collapse of the gas in a thin disk, i.e. a "created on the spot" thick disk. We also notice the Samland (2004) scenario: a chemodynamical model of formation of a disk galaxy within a growing dark halo that provides both a "cold" thick disk and a metal-poor "hot" thick disk.

A popular scenario is the "accreted" thick disk formed from the accretion of satellites. If the thick disk results from the accretion of just a single satellite, with a fifth of the mass of the Galactic disk, this has been certainly a major event in the history of the Galaxy, and it is hard to believe that the thin disk could have survived this upheaval.

Finally, from the thick disk properties identified in this paper, we can reject the most improbable scenario of formation: the one of type "heated" thick disk (by molecular clouds or spiral arms).

Acknowledgements. Funding for RAVE has been provided by the AngloAustralian Observatory, by the Astrophysical Institute Potsdam, by the Australian Research Council, by the German Research foundation, by the National Institute for Astrophysics at Padova, by The Johns Hopkins University, by the Netherlands Research School for Astronomy, by the Natural Sciences and Engineering Research Council of Canada, by the Slovenian Research Agency, by the Swiss National Science Foundation, by the National Science Foundation of the USA (AST-0508996), by the Netherlands Organisation for Scientific Research, by the Particle Physics and Astronomy Research Council of the UK, by Opticon, by Strasbourg Observatory, and by the Universities of Basel, Cambridge, and Groningen. The RAVE web site is at http://www . rave-survey.org.

Data verification is partially based on observations taken at the Observatoire de Haute Provence (OHP, France), operated by the French CNRS.

This publication makes use of data products of the 2MASS, which is a joint project of the University of Massachusetts and the Infrared Processing and Analysis Center, funded by the NASA and NSF

It is a pleasure to thank the UCAC team who supplied a copy of the UCAC CD-ROMs in July 2003.

This research has made use of the SIMBAD and VIZIER databases, operated at CDS, Strasbourg, France.

This paper is based on data from the ESA HIPPARCOS satellite (HIPPARCOS and TYCHO-II catalogues).

\section{References}

Abadi, M. G., Navarro, J. F., Steinmetz, M. 2003, ApJ, 597, 21

Alves, D. 2000, ApJ, 539, 732

Bahcall, J. N. 1984, ApJ, 287, 926

Bartašiūtė, S. 1994, Balt. Astr., 3, 16

Bienaymé, O. 1999, A\&A, 341, 86

Bienaymé, O., \& Séchaud, N. 1997, A\&A, 323, 781

Bienaymé, O., Robin, A. C., \& Crézé, M. 1987, A\&A, 186, 359

Bienaymé, O., Soubiran, C., Mishenina, T. V., Kovtyukh, V. V., \& Siebert, A. 2006, A\&A, 446, 933

Blaauw, A. 1995, Stellar populations, ed. P. C. Van der Kruit, \& G. Gilmore (Dordrecht: Kluwer Academic Publishers), IAU Symp., 164, 39 Brewer, M.-M., \& Carney, B. 2006, ApJ, 131, 431

Brook, C., Kawata, D., Gibson, B., \& Freeman, K. 2004, ApJ, 612, 894

Cabrera-Lavers, A., Garzón, F., \& Hammersley, P. L. 2005, A\&A, 433, 173

Cannon, R. D. 1970, MNRAS, 150, 111

Carraro, G., Ng, Y. K., \& Portinari, L. 1998, MNRAS, 296, 1045

Chiba, M., \& Beers, T. 2000 ApJ, 119, 2843

Cubarsi, R., \& Alcobé, S. 2004, A\&A, 427, 131
Cutri, R., et al. 2003, The IRSA 2MASS All-Sky Point Source Catalog, NASA/IPAC Infrared Science Archive http: //irsa.ipac . caltech. edu/ applications/Gator/

Dehnen, W., \& Binney, J. 1998, MNRAS, 298, 387

Ducati, J., Bevilacqua, C., Rembold, S., \& Ribeiro, D. 2001, ApJ, 555, 309

Ducourant, C., Le Campion, J. F., Rapaport, M., et al. 2006, A\&A, 448, 1235

Edvardsson, B., Andersen, J., Gustafsson, B., et al. 1993, A\&A, 275, 101

Feltzing, S., Bensby, T., \& Lundström, I. 2003, A\&A, 397, L1

Famaey, B., Jorissen, A., Luri, X., et al. 2005a, A\&A, 430, 165

Famaey, B., Jorissen, A., \& Luri, X. 2005b, Proc. of the Gaia Symposium The Three-Dimensional Universe with Gaia (ESA SP-576), ed. C. Turon, et al., 129

Flynn, C., \& Morell, O. 1997, MNRAS, 286, 617

Fuhrmann, K. 1998, A\&A, 338, 161

Gilmore, G., \& Reid, N. 1983, MNRAS, 202, 1025

Gilmore, G., \& Wyse, R. 1985, AJ, 90, 2015

Gilmore, G., Wyse, R., \& Kuijken, K. 1989, ARA\&A, 27, 555

Gilmore, G., Wyse, R., \& Jones, J. B. 1995, 109, 1095

Gilmore, G., Wyse, R., \& Norris, J. 2002, ApJ, 574, L39

Girardi, L., Groenewegen, M., Weiss, A., \& Salaris, M. 1998, MNRAS, 301, 149

Girardi, L., Bertelli, G., Bressan, A., et al. 2002, A\&A, 391, 195.

Girardi, L., Groenewegen, M. A. T., Hatziminaoglou, E., \& da Costa, L. 2005, A\&A, 436, 895

Gould, A. 2003, ApJ, 583, 765

Grocholski, A., \& Sarajedini, A. 2002, AJ, 123, 1612

Holmberg, J., \& Flynn, C. 2004, MNRAS, 352, 440

Iovino, A., McCracken, H. J., Garilli, B., et al. 2005, A\&A, 442423

James, F. 2004, MINUIT Tutorial from 1972 CERN Computing and Data Processing School

Koornneef, J. 1983, A\&A, 128, 84

Kotoneva, E., Flynn, C., \& Jimenez, R. 2002, MNRAS, 335, 1147

Kuijken, K., \& Gilmore, G., MNRAS, 239, 605

Lewis, J. R., \& Freeman, K. C. 1989, AJ, 97, 139

Majewski, S. R. 1993, ARA\&A, 31, 575

Martin, J. C., \& Morrison, H. L. 1998, AJ, 116, 1724

Morrison, H. L., Flynn, C., \& Freeman, K. C. 1990, AJ, 100, 1191

Navarro, J. F., Helmi, A., Freeman, K. C., et al. 2004, ApJ, 601, L43

Nordström, B., Mayor, M., Andersen, J., et al. 2004, A\&A, 418, 989

Norris, J., Bessell, M. S., \& Pickles, A. J., 1985, ApJS, 58, 463

Ojha, D. 2001, MNRAS, 322, 426

Oort, J. H. 1922, Bull. Astron. Inst. Netherlands, 1, 133

Pauli, E.-M., Heber, U., Napiwotzki, R., et al. 2005, 4th European Workshop on White Dwarfs, ed. D. Koester, \& S. Moehler, ASP Conf. Ser., 334, 81

Pichon, C., Siebert, A., \& Bienaymé, O. 2002, MNRAS, 329, 181

Pritchet, C. 1983, AJ, 88, 1476

Quinn, P.J., Hernquist, L., \& Fullagar, D. P. 1993, ApJ, 403, 74

Ratnatunga, K., Bahcall, J., \& Casertano, S. 1989, ApJ, 339, 106

Reddy, B. E. \& Lambert, D. L., \& Allende Prieto, C. 2006, MNRAS, 367, 1329

Reid, I. N. 1998, AJ, 115, 204

Robin, A. C., \& Crézé, M. 1986, A\&A, 157, 71

Robin, A. C., Reylé, C., Derrière, S., \& Picaud, S. 2003, A\&A, 409, 523

Robin, A. C., Reylé , Derrière, S., \& Picaud, S. 2004, A\&A, 416, 157

Soubiran, C., \& Girard, P. 2005, A\&A, 438, 139

Soubiran, C., Bienaymé, O., \& Siebert, A. 2003, A\&A, 398, 141

Samland, M. 2004, PASA, 21, 175

Samland, M., \& Gerhard, O. 2003, A\&A, 399, 961

Salaris, M., \& Girardi, L. 2002, MNRAS, 337, 332

Sarajedini, A. 2004 AJ, 128, 1228

Steinmetz, M., \& Navarro, J. 2002, New A., 7, 155

Spagna, A., Lattanzi, M. G., Lasker, B. M., et al. 1996, A\&A, 311, 758

Steinmetz, M. 2003, GAIA Spectroscopy: Science and Technology, held 9-12 September 2002 at Gressoney St. Jean, Aosta, ed. U. Munari, ASP Conf. Proc., 298, 381

Steinmetz, M., Zwitter, T., Siebert, A., et al. 2006, AJ, 132, 1645

Vallenari, A., Pasetto, S., Bertelli, G., Chiosi, C., Spagna, A., \& Lattanzi, M. 2006, A\&A, 451, 125

van der Kruit, P. C., \& Searle, L. 1982, A\&A, 110, 61

Wainscoat, R. J., Cohen, M., Volk, K., Walker, H. J., \& Schwartz, D. E. 1992, ApJS, 83, 111

Wyse, R., Gilmore, G., Norris, J., et al. 2006, ApJ, 639, L13

Zacharias, N., Urban, S. E., Zacharias, M. I., et al. 2004, AJ, 127, 3043

Zwitter, T., et al. 2008, AJ, submitted 